\title{
Propagation dynamics and $\mathrm{X}$-pulse formation in phase-mismatched second-harmonic generation
}

\author{
G. Valiulis,,${ }^{1, *}$ V. Jukna, ${ }^{1}$ O. Jedrkiewicz, ${ }^{2}$ M. Clerici, ${ }^{2}$ E. Rubino, ${ }^{2}$ and P. DiTrapani ${ }^{2}$ \\ ${ }^{1}$ Department of Quantum Electronics, Vilnius University, Sauletekio 9, Bldg. 3, LT-10222 Vilnius, Lithuania \\ ${ }^{2}$ CNISM and Dipartimento di Fisica e Matematica, University of Insubria, Via Valleggio 11, IT-22100 Como, Italy
}

(Received 2 December 2010; published 28 April 2011)

\begin{abstract}
This paper concerns the theoretical, numerical, and experimental study of the second-harmonic-generation (SHG) process under conditions of phase and group-velocity mismatch and aims to demonstrate the dimensionality transition of the SHG process caused by the change of the fundamental wave diameter. We show that SHG from a narrow fundamental beam leads to the spontaneous self-phase-matching process with, in addition, the appearance of angular dispersion for the off-axis frequency components generated. The angular dispersion sustains the formation of the short $\mathrm{X}$ pulse in the second harmonic $(\mathrm{SH})$ and is recognized as three-dimensional (3D) dynamics. On the contrary, the large-diameter fundamental beam reduces the number of the degrees of freedom, does not allow the generation of the angular dispersion, and maintains the so-called one-dimensional (1D) SHG dynamics, where the self-phase-matching appears just for axial components and is accompanied by the shrinking of the SH temporal bandwidth, and sustains a long SH pulse formation. The transition from long SH pulse generation typical of the 1D dynamics to the short 3D X pulse is illustrated numerically and experimentally by changing the conditions from the self-defocusing to the self-focusing regime by simply tuning the phase mismatch. The numerical and experimental verification of the analytical results are also presented.
\end{abstract}

DOI: 10.1103/PhysRevA.83.043834

PACS number(s): 42.65.Sf, 42.65.Ky, 42.65.Re, 42.79.Nv

\section{INTRODUCTION}

Since 1961, when Franken and his collaborators experimentally discovered second-harmonic generation (SHG) [1], the process of SHG has been widely investigated leading first to important contributions by Refs. [2-6], who derived the now well-known theory described in nonlinear optics books. It was also realized that the use of short light pulses for second-harmonic generation gives rise to special effects such as the broadening of the generated light pulse due to the group-velocity difference between the fundamental harmonic (FH) frequency and the second-harmonic ( $\mathrm{SH})$ frequency pulses, predicted by Comly and co-workers [7] and observed by Shapiro [8].

Typically, SHG is investigated or used at or near the phase-matching condition in such a way as to maximize the conversion efficiency. More recently, the study of propagation phenomena in phase-mismatched second-harmonic processes has attracted considerable interest, in particular for the investigation of the effect on the generated pulse shape. In 1969, Glenn provided a general solution of the SH field, which showed two contributions, one arising as a surface term, traveling with the characteristic group velocity expected at the SH frequency, and a second component that instead appeared to travel with the velocity of the FH beam [9]. The combined effect of phase-mismatch and group-velocity difference was studied both analytically $[10,11]$ and in combination with depletion numerically [12], showing the occurrence of a double-peak structure in the SH signal. This feature was also shown to arise as a result of group-velocity dispersion within the bandwidth of the fundamental pulse [13]. In particular, the

\footnotetext{
*Corresponding author; gintaras.valiulis@ff.vu.lt
}

predicted pulse splitting of the second-harmonic signal due to the group-velocity difference between the fundamental and the generated second harmonic was observed experimentally by Noordam and co-workers [14].

The modification of the temporal and spectral shapes of the pulses generated in SHG processes has also been analyzed experimentally by looking at the influence of cascading selfphase and cross-phase modulation [15]. Theoretical aspects of such phenomena have also been discussed beyond the regime of the usual slowly varying envelope approximation by Mlejnek et al. in [16] for the general case of nonlinear dielectrics, and by $\mathrm{Su}$ et al. [17] in BBO crystals both theoretically and experimentally.

Recently, the generation of two distinct components in the conditions of phase and group-velocity mismatch and low pump intensities was interpreted as a phase-locking mechanism that causes the trapping of portions of the $\mathrm{SH}$ signal, which then travel under the pump pulse [18], as shown experimentally in absorbing materials [19]. Lately, the phaseand group-velocity locking of $\mathrm{FH}$ and $\mathrm{SH}$ pulses was also observed at oblique incidence in a lithium niobate crystal [20].

Phase-mismatched SHG processes in the presence of large group-velocity mismatch (GVM) have also been investigated in the context of applications. Namely, Marangoni and coworkers have demonstrated a simple method for the generation of tunable picosecond pulses synchronized with femtosecond pulses [21]. To this end, they exploit the SHG process in this regime to efficiently transfer the energy of a broadband FH pulse into a narrowband SH pulse, thus allowing us to obtain tunable picosecond pulses, as also shown in [22]. The narrow-bandwidth picosecond pulses were also generated with predetermined spectral and temporal shapes, as recently demonstrated by means of quasi-phase-matched nonlinear structures [23]. 
The so-called nonlinear X waves were observed in phasemismatched SHG processes [24,25] (recently also in a different regime in [26]), as well as investigated in Kerr media [27-30]. The nonlinear $\mathrm{X}$ waves are weakly localized nondiffracting and nondispersing wave packets characterized by a nonseparable spatiotemporal profile (propagation invariant solutions) [31,32], accessible in the normal group-velocity dispersion regime, the formation of which is triggered by conical emission [33]. In [34], it was theoretically demonstrated that an X wave at $\mathrm{SH}$ can be generated spontaneously by doubling a broad FH pump beam in the undepleted stage of its propagation. Moreover, as shown in [24], in SHG, these wave packets are constituted by X-shaped envelopes at the $\mathrm{SH}$ and $\mathrm{FH}$ frequency traveling locked together.

This paper is aimed at highlighting the transition between the one-dimensional (1D) case, where diffraction is negligible, and the three-dimensional (3D) case, where diffraction and angular dispersion become important in the SHG process, especially in relation to the reshaping of the $\mathrm{SH}$ pulses involved. Thus, we will present a clear and detailed analysis of the propagation dynamics occurring in phase-mismatched secondharmonic-generation processes, corroborated by numerical simulations in various regimes of focusing, beam dimensions, positive or negative phase mismatch, presence or absence of group-velocity mismatch, group-velocity dispersion, and in relation with the spontaneous $\mathrm{SH} \mathrm{X}$-wave formation. Experimental results performed in the femtosecond-pulsed regime in different parameter cases in a lithium triborate crystal (LBO) will also be presented. The paper is organized as follows. In Sec. II, the description of self-phase matching in SHG is presented in the one- and three-dimensional cases, with an illustration of several interesting regimes (also from the numerical point of view). In Sec. III, the transition from the $1 \mathrm{D}$ to the $3 \mathrm{D}$ cases is discussed and demonstrated by means of numerical simulations, while experimental results are presented in Sec. IV. Conclusions are presented in Sec. V.

\section{SELF-PHASE MATCHING IN SHG}

In this section, we discuss the main features of the phasemismatched SHG process by means of an analytical and a numerical study. The collinear SHG in the second-order dispersion approximation and in the reference frame moving with the group velocity of the fundamental harmonic wave is described by the set of equations for the interacting fields envelopes given by

$$
\begin{array}{r}
\frac{\partial A_{1}}{\partial z}-\frac{i}{2} g_{1} \frac{\partial^{2} A_{1}}{\partial t^{2}}+\frac{i}{2 k_{10}} \Delta_{\perp} A_{1}=-\sigma_{1} A_{1}^{*} A_{2} e^{-i \Delta k z} \\
\frac{\partial A_{2}}{\partial z}+v_{21} \frac{\partial A_{2}}{\partial t}-\frac{i}{2} g_{2} \frac{\partial^{2} A_{2}}{\partial t^{2}}+\frac{i}{2 k_{20}} \Delta_{\perp} A_{2}=\sigma_{2} A_{1}^{2} e^{i \Delta k z}
\end{array}
$$

where $A_{j}(t, x, y, z)$ is the complex amplitude, $t$ is the retarded time (corresponding to the coordinate frame moving with the fundamental wave), $x$ and $y$ are transverse coordinates, and $z$ is the longitudinal coordinate, respectively. The indexes $j=1,2$ stand for fundamental and second-harmonic waves, respectively. $v_{i j}=1 / u_{i}-1 / u_{j}$ is the group-velocity mismatch, $u_{j}$ is the group velocity of wave $j$ calculated at carrier frequency $\omega_{j 0}$, and $\omega_{20}=2 \omega_{10}$. Parameters $g_{j}(i, j=1,2)$ are the group-velocity dispersion (GVD) coefficients for the $\mathrm{FH}$ and SH waves. The parameter $k_{j 0}=\left|\mathbf{k}_{\mathbf{j 0}}\right|$ is the modulus of the wave $j$ axial wave vector (both $\mathrm{FH}$ and $\mathrm{SH}$ are collinear to the propagation axis $z$ ) calculated at the carrier frequencies. $\Delta k=k_{20}-2 k_{10}$ is the phase mismatch along the propagation axis calculated at the carrier frequency, $\sigma_{j}$ is the nonlinear coupling coefficient, and $\Delta_{\perp}$ is the transverse Laplace operator.

In the case of the undepleted SHG process, we can write the set of Eq. (1) by introducing new amplitudes $B_{1}=A_{1}$ and $B_{2}=A_{2} \exp (-i \Delta k z)$ :

$$
\begin{gathered}
\frac{\partial B_{1}}{\partial z}-\frac{i}{2} g_{1} \frac{\partial^{2} B_{1}}{\partial t^{2}}+\frac{i}{2 k_{10}} \Delta_{\perp} B_{1}=0, \\
\frac{\partial B_{2}}{\partial z}+v_{21} \frac{\partial B_{2}}{\partial t}-\frac{i}{2} g_{2} \frac{\partial^{2} B_{2}}{\partial t^{2}}+\frac{i}{2 k_{20}} \Delta_{\perp} B_{2}+i \Delta k B_{2}=\sigma_{2} B_{1}^{2} .
\end{gathered}
$$

The fundamental wave dynamics is governed by a linear equation that can be solved independently from the equation for the SH. In general, the fundamental wave amplitude has a temporal and spatial profile affected by the medium dispersion and by diffraction, thus, in general, $B_{1}=B_{1}(t, x, y, z)$. We will assume below that the fundamental wave is undepleted, undistorted, and propagates with group velocity $u_{1}$ without changing its spatial and temporal profile, thus, in our reference frame $B_{1}=B_{1}(t, x, y)$. The investigation of the SHG process will be performed in one-dimensional (plane-wave model) as well as in three-dimensional (pulsed-beam model) approximations. Indeed, in the following, we shall present an in-depth investigation of the SHG process in different regimes, starting from the one-dimensional case and then showing the impact of the space-time coupling on those regimes in the three-dimensional case. Despite the fact that some of the cases discussed in the following relative to one-dimensional SHG were studied by various authors (as mentioned in the Introduction), here we will present some classification of the SHG regimes based on the $\mathrm{SH}$ wave decomposition into several components. This is necessary and has the purpose of demonstrating the differences and similarities between oneand three-dimensional cases and revealing the possibilities of the X-wave formation in $\mathrm{SH}$.

\section{A. One-dimensional SHG}

We start here by restricting ourselves to the plane-wave approximation (assuming large beam diameters), so that, in Eq. (2), the term with the Laplace operator can be neglected. We also assume a negligible group-velocity dispersion of the fundamental wave, so $B_{1}=B_{1}(t)$. Under these assumptions, the second equation of Eq. (2) becomes

$$
\frac{\partial B_{2}}{\partial z}+v_{21} \frac{\partial B_{2}}{\partial t}-\frac{i}{2} g_{2} \frac{\partial^{2} B_{2}}{\partial t^{2}}+i \Delta k B_{2}=\sigma_{2} B_{1}^{2} .
$$

We look for the solution of Eq. (3) in the Fourier space by introducing the spectral representation for the $\mathrm{SH}$ and $\mathrm{FH}$ waves, respectively,

$$
B_{2}(t, z)=\frac{1}{2 \pi} \int_{-\infty}^{\infty} S(\Omega) \exp (i \Omega t) d \Omega,
$$




$$
B_{1}^{2}(t)=\frac{1}{2 \pi} \int_{-\infty}^{\infty} F(\Omega) \exp (i \Omega t) d \Omega .
$$

Note that the SHG in the undepleted approximation is governed just by the squared envelope of the FH wave, so if we are not interested in the FH dynamics, having the function $F(\Omega)$ instead of the fundamental wave spectrum is enough. After substitution of Eqs. (4) and (5) into (3), we obtain

$$
\frac{\partial S}{\partial z}+i D(\Omega) S=\sigma_{2} F(\Omega)
$$

where we define

$$
D(\Omega)=\Delta k+v_{21} \Omega+\frac{1}{2} g_{2} \Omega^{2} .
$$

The solution of Eq. (6) with the zero SH condition at the input $[S(\Omega, z=0)=0]$ is

$$
S(\Omega, z)=z \sigma_{2} F(\Omega) \operatorname{sinc}\left(\frac{D(\Omega) z}{2}\right) \exp \left(-i \frac{D(\Omega) z}{2}\right) .
$$

The key parameter describing the SHG dynamics is $D(\Omega)$. Note that $\Omega$ is the absolute $\omega$ frequency detuning from the SH carrier $\left(\Omega=\omega-\omega_{20}\right)$. The most effective second-harmonicgeneration process can be obtained if $D(\Omega)=0$. In this case, the SH wave sustains a stationary profile with amplitude growing linearly with the propagation distance $z[S(\Omega, z)=$ $\left.z \sigma_{2} F(\Omega)\right]$. It is clear that, due to material dispersion, the condition $D(\Omega)=0$ can not be satisfied for all frequency ranges. This makes the frequency band for SHG defined by the product of the "squared" fundamental wave spectrum $F(\Omega)$ and the function $\operatorname{sinc}\left(\frac{D(\Omega) z}{2}\right)$.

For a better interpretation of the main features of the obtained solution, we rewrite Eq. (8) as

$$
S(\Omega, z)=i \sigma_{2} \frac{F(\Omega)}{D(\Omega)}(\exp \{-i[D(\Omega) z]\}-1),
$$

showing how the SH wave may be described as composed by a free and a driven wave as

$$
S(\Omega, z)=S^{(f)}(\Omega, z)+S^{(d)}(\Omega) .
$$

The free wave is the solution of the homogeneous equation $\left[\frac{\partial S}{\partial z}+i D(\Omega) S=0\right]$, which describes linear wave-packet propagation at carrier frequency $\omega_{20}$ and is given by

$$
S^{(f)}(\Omega, z)=i \sigma_{2} \frac{F(\Omega)}{D(\Omega)} \exp [-i D(\Omega) z] .
$$

The driven wave solution is constant along the propagation distance $z$ and satisfies Eq. (6):

$$
S^{(d)}(\Omega)=-i \sigma_{2} \frac{F(\Omega)}{D(\Omega)} .
$$

Note that the reference frame is fixed with the fundamental wave, thus, the driven $\mathrm{SH}$ propagates with the group velocity of the $\mathrm{FH}$, that is, $u_{1}$ and the free $\mathrm{SH}$ wave due to phase shift $\exp [-i D(\Omega) z]\}$ propagates with a group velocity $u_{2}$ (as for a pulse of carrier frequency $\omega_{20}$ in a linear medium).

We should note that the obtained solutions could be extended to the case of linearly absorbing media. In that case, the left side of Eq. (3) contains the additional term $\alpha B_{2}$ accounting for the linear losses, where $\alpha>0$ is the linear absorption coefficient. Therefore, the solution has the same form as before, but the quantity $D(\Omega)$ becomes complex and can now be written as

$$
D(\Omega)=-i \alpha+\Delta k+v_{21} \Omega+\frac{1}{2} g_{2} \Omega^{2} .
$$

We observe that, when $z \gg 1 / \alpha$, the free wave vanishes and the SH contains only the driven component with constant amplitude $S(\Omega, z) \approx S^{(d)}(\Omega)$ (despite linear losses).

We present in the following the discussion of different cases.

Case A: The fundamental wave nearly monochromatic $\left[F(\Omega) \approx 2 \pi F_{0} \delta(\Omega)\right]$ [here $\delta(\Omega)$ is the Dirac delta function], or material dispersion (GVM, GVD) negligible $\left(v_{21} \rightarrow 0, g_{2} \rightarrow\right.$ $0)$ in the case of phase-mismatched $(\Delta k \neq 0)$ SHG. We have $D(\Omega)=\Delta k$ and, thus, by means of Eqs. (4) and (8), we find

$$
B_{2}(t, z)=\frac{2 \sigma_{2} F_{0}}{\Delta k} \sin \left(\frac{\Delta k z}{2}\right) \exp \left(-i \frac{\Delta k z}{2}\right) .
$$

The intensity of the second-harmonic $\left|B_{2}\right|^{2} \propto \sin ^{2}\left(\frac{\Delta k z}{2}\right)$ exhibits well-known oscillations versus $z$ as the result of the spatial beating between the free and the driven SH waves, as discussed in every handbook of nonlinear optics (for example, see [35]). Note that in the dissipative case, when $z \gg 1 / \alpha$, the SH field reduces to the driven wave with constant amplitude

$$
B_{2}(t, z)=\frac{\sigma_{2}}{\alpha+i \Delta k} F_{0} .
$$

Case B: Phase-matched SHG with GVM dominant over GVD $\left(\Delta k=0, v_{21} \neq 0, g_{2} \cong 0\right)$. In this case, we have $D(\Omega) \approx v_{21} \Omega$ and the solution of Eq. (8) becomes

$S(\Omega, z)=z \sigma_{2} F(\Omega) \operatorname{sinc}\left(\frac{\nu_{21} \Omega z}{2}\right) \exp \left(-i \frac{\nu_{21} \Omega z}{2}\right)$.

The spectral width of the function $\operatorname{sinc}\left(\frac{v_{21} \Omega z}{2}\right)$ (defined as the interval between the first two zeros around the value $\Omega=$ $0)$ decreases with the distance as $\Delta \Omega_{\text {sinc }}=4 \pi /\left(\nu_{21} z\right)$. If the FH bandwidth [width of the function $F(\Omega)$ ] is $\Delta \Omega_{\mathrm{FH}}$, for $z \gg 4 \pi /\left(v_{21} \Delta \Omega_{\mathrm{FH}}\right)$, we have $\Delta \Omega_{\text {sinc }} \ll \Delta \Omega_{\mathrm{FH}}$ and

$$
S(\Omega, z) \approx z \sigma_{2} F(0) \operatorname{sinc}\left(\frac{\nu_{21} \Omega z}{2}\right) \exp \left(-i \frac{\nu_{21} \Omega z}{2}\right) .
$$

The above equation describes the spectrum of a rectangular pulse with duration $\tau=\left|\nu_{21} z\right|$, thus growing proportionally to the propagation distance $z$. Therefore, the phase-matched SHG process with large GVM supports, via spectral filtering (i.e., shrinking of the sinc function), the formation of a nearly rectangular $\mathrm{SH}$ pulse with such a duration. Note that, in the limit case $z \rightarrow \infty$, we obtain a monochromatic wave since the spectra is described by the Dirac delta function $\left[\lim _{z \rightarrow \infty} \frac{\sin (\Omega z)}{\pi \Omega}=\delta(\Omega)\right]$. Moreover, from Eq. (9), we find that the leading and trailing fronts of the SH pulse are propagating with different velocities, corresponding to the fact that the rectangular SH pulse is made of two waves: the free component propagating with velocity $u_{2}$ and the driven one propagating with velocity $u_{1}$. In the dissipative medium, for $z \gg 1 / \alpha$, only the driven $\mathrm{SH}$ wave survives:

$$
S(\Omega, z) \approx \frac{\sigma_{2}}{\alpha+i \nu_{21} \Omega} F(\Omega) .
$$


Note that $S(\Omega, z) \propto F(\Omega)$ and the temporal profile of such a wave is similar to the square envelope of the $\mathrm{FH}$, namely $B_{2} \propto B_{1}^{2}$ [with some asymmetry due to the fractional term in Eq. (18)].

The analytical results presented here have been reproduced by numerical simulations by means of a split-step code solving the system of Eqs. (1) and accounting for the medium parameters.

The group-velocity-mismatch parameter $v_{21}$ was taken into account via the group-velocity-mismatch length $L_{v}=$ $\tau_{0} / \nu_{21}$ calculated for the $\tau_{0}=1$ ps pulse; the groupvelocity-dispersion parameter $g_{j}$ via the GVD length $L g_{j}=$ $\tau_{0}^{2} /\left(4 \ln 2 g_{j}\right)$, which defines the distance over which the duration of the Gaussian $\tau_{0}=1$ ps pulse increases by a factor of $\sqrt{2}$. The nonlinearity of the medium was accounted for by introducing the nonlinear length $L_{n}=\left(\sigma_{2} A_{0}\right)^{-1}$, where $A_{0}$ is the pulse amplitude corresponding to the pulse intensity of $1 \mathrm{GW} / \mathrm{cm}^{2}$. The linear losses parameter $\alpha$ for the $\mathrm{SH}$ was taken into account through the absorption length $L_{\alpha}=(2 \alpha)^{-1}$, defined as the distance over which the intensity drops by factor of $e$, due to absorption. The temporal envelopes and the spectral profiles of the interacting pulses obtained from the numerical solution of Eq. (1) in Case B are presented in Fig. 1. The artificial (with made-up dispersion and nonlinearity) $L=$ $30 \mathrm{~mm}$ crystal with switched-off GVD $\left(L g_{j}=\infty\right)$ and typical (for most real crystals) other parameters $L_{v}=25 \mathrm{~mm}, L_{n}=1$ $\mathrm{mm}$ was used. The fundamental pulse full width at half maximum (FWHM) duration was set to $\tau=0.1 \mathrm{ps}$, intensity $I_{\mathrm{FH}}=10 \mathrm{MW} / \mathrm{cm}^{2}$, and $\Delta k=0 \mathrm{~cm}^{-1}$. When absorption is negligible, the short FH pulse [see Fig. 1(a), dashed line] generates a broad SH pulse [Fig. 1(a), solid line] with the temporal bandwidth that shrinks [Fig. 1(c)]. If the linear absorption is significant $\left(L_{\alpha}=2 \mathrm{~mm}\right)$, only the driven $\mathrm{SH}$ wave
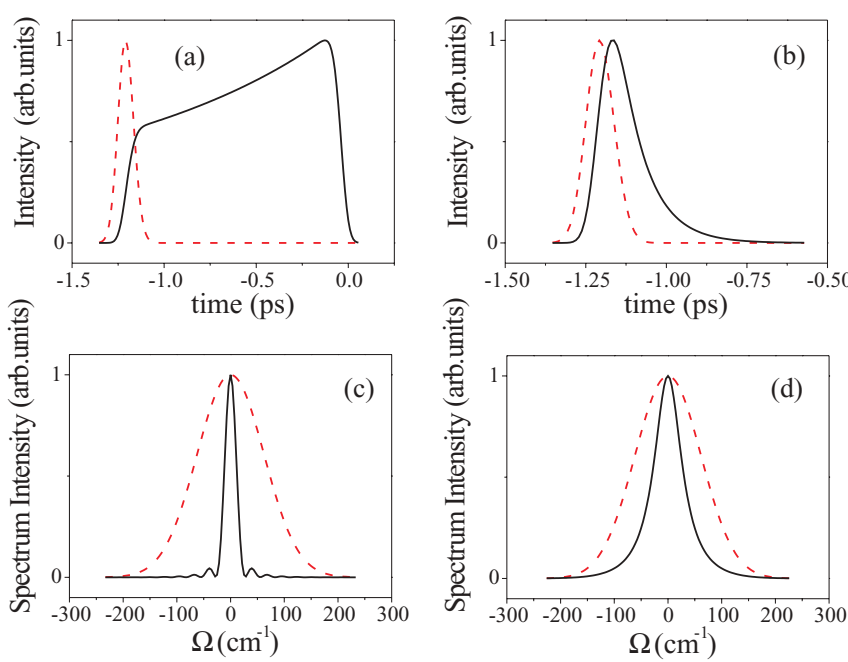

FIG. 1. (Color online) Numeric demonstration of Case B. The SH (solid line) and FH (dashed line) (a), (b) intensity and (c), (d) spectra profiles at the exit of the nonlinear medium presented. The left column (a), (c) depicts the case without and the right column (b), (d) depicts the case with linear losses. The parameters used (see text) are $L=30 \mathrm{~mm}, L_{v}=25 \mathrm{~mm}, L_{n}=1 \mathrm{~mm}, \tau=0.1 \mathrm{ps}$, $I_{\mathrm{FH}}=10 \mathrm{MW} / \mathrm{cm}^{2}$, and $\Delta k=0$. For the dissipative case (b), (d), $L_{\alpha}=2 \mathrm{~mm}$. survives sustaining a short SH pulse formation [Figs. 1(b) and 1(d)], in agreement with the above-discussed solution (18). We mention that originally the effects of the pulse broadening were predicted by Comly and co-workers [7] and observed by Shapiro [8] and also observed later in quasi-phase-matching structures by Marangoni and co-authors [21,23]. The role of the linear absorption was also studied in [19].

Case $C$ : Phase-mismatched SHG with GVM dominant over GVD $\left(\Delta k \neq 0, v_{21} \neq 0, g_{2} \cong 0\right)$. In this case, $D(\Omega) \approx \Delta k+$ $v_{21} \Omega$ and therefore the dynamics depends on the FH pulse bandwidth. Two possible subcases can be analyzed.

(i) The first one is when the bandwidth of the $\mathrm{FH}$ is so narrow that it does not contain frequencies around $\Omega_{0}=-\frac{\Delta k}{v_{21}}$, where $D\left(\Omega_{0}\right)=0$. Thus, in proximity to the carrier frequency $(\Omega \approx 0)$, we have $D(\Omega) \approx \Delta k$ and the solution described by Eqs. (10)-(12) reads as

$$
\begin{gathered}
S^{(f)}(\Omega, z) \approx i \frac{\sigma_{2}}{\Delta k} F(\Omega) \exp \left[-i\left(\Delta k+v_{21} \Omega\right) z\right], \\
S^{(d)}(\Omega) \approx-i \frac{\sigma_{2}}{\Delta k} F(\Omega) .
\end{gathered}
$$

Here, the $\mathrm{SH}$ wave is composed by two pulses with nearly equal profile [given by the function $F(\Omega)$ that corresponds to the square FH temporal envelope]. The exponential term $\exp \left(-i v_{21} \Omega z\right)$ in Eq. (19) corresponds to a temporal shift of the pulse envelope by a quantity $T=v_{21} z$. Therefore, instead of a broad pulse as in Case B, the SH generated contains two pulses with durations nearly equal to that of the FH pulse but propagating with different group velocities: the free $\mathrm{SH}$ component [Eq. (19)] propagates with group velocity $u_{2}$ and the driven component [Eq. (20)] with group velocity $u_{1}$. In the linearly absorbing medium [instead of $\Delta k$ we should substitute $\Delta k-i \alpha$ in Eq. (19)] when $z \gg 1 / \alpha$, only the driven wave with constant amplitude survives and, from Eq. (20), we have $B_{2}(t, z) \approx-i \frac{\sigma_{2}}{\Delta k} B_{1}^{2}(t)$.

(ii) The second scenario occurs when the spectrum of the $\mathrm{FH}$ contains frequencies close to $\Omega_{0}=-\frac{\Delta k}{v_{21}}$, therefore, the condition $D(\Omega) \approx 0$ becomes valid (at the proximity of $\Omega_{0}$ ), and the dynamics described in Case B becomes possible at a shifted frequency: a new SH component with nearly rectangular envelope appears at a carrier frequency shifted by $\Omega_{0}$. Moreover, this new component may be significant even if $F\left(\Omega_{0}\right) \ll F(\Omega=0)$. This becomes possible because of the fulfillment of the condition $\left.D(\Omega)\right|_{\Omega \rightarrow \Omega_{0}} \rightarrow 0$. For a quantitative description, the solution described by Eq. (9) can be rewritten in these conditions as the sum of two components

$$
S(\Omega, z)=S^{(1,2)}(\Omega, z)+S^{(3)}(\Omega, z)
$$

where

$$
\begin{gathered}
S^{(1,2)}(\Omega, z)=i \sigma_{2} \frac{F(\Omega)-F\left(\Omega_{0}\right)}{D(\Omega)}\{\exp [-i D(\Omega) z]-1\}, \\
S^{(3)}(\Omega, z)=z \sigma_{2} F\left(\Omega_{0}\right) \operatorname{sinc}\left(\frac{D(\Omega) z}{2}\right) \exp \left(-i \frac{D(\Omega) z}{2}\right) .
\end{gathered}
$$

After introducing the frequency shift $\Omega_{0}$, we have $D(\Omega)=$ $\nu_{21}\left(\Omega-\Omega_{0}\right)$ and the component $S^{(3)}$ becomes equal to

$S^{(3)}=z \sigma_{2} F\left(\Omega_{0}\right) \operatorname{sinc}\left(\frac{\nu_{21}\left(\Omega-\Omega_{0}\right) z}{2}\right) e^{-i \frac{\nu_{21}\left(\Omega-\Omega_{0}\right) z}{2}}$. 
Equation (24) describes the spectrum of a rectangular pulse with carrier frequency shifted by $\Omega_{0}$ and duration $\left(\tau=\left|\nu_{21}\right| z\right)$ growing proportionally to the propagation distance $z$. On the contrary, the component $S^{(1,2)}$ has no influence on the SH wave at frequency $\Omega_{0}$, but around the carrier frequency $(\Omega \approx 0)$ we have $D(\Omega) \approx \Delta k$. Thus, $S^{(1,2)}$ describes a wave composed by two pulses with different velocities [see Eqs. (9)-(12)]. The temporal envelope of such pulse is defined by the spectrum $F(\Omega)-F\left(\Omega_{0}\right)$. If the carrier component dominates, $|F(0)| \gg$ $\left|F\left(\Omega_{0}\right)\right|$ and, thus, for the spectral components around $\Omega=0$, we obtain from Eq. (22)

$$
\begin{aligned}
S^{(1,2)}(\Omega, z) & \approx i \frac{\sigma_{2}}{\Delta k} F(\Omega)\left\{\exp \left[-i\left(\Delta k+v_{21} \Omega\right) z\right]-1\right\} \\
& =\operatorname{const}_{1} F(\Omega) \exp \left(-i \nu_{21} \Omega z\right)+\operatorname{const}_{2} F(\Omega) .
\end{aligned}
$$

Equation (25) describes two pulses with the same envelope $\sim B_{1}^{2}(t)$ separated in time by an interval $T=\left|v_{21} z\right|$, i.e., the pulses are propagating with different velocities $\left[u_{1}=\right.$ $\left.u\left(\omega_{10}\right), u_{2}=u\left(\omega_{20}\right)\right]$. We can say, therefore, that in the case of large $\mathrm{FH}$ bandwidth, the $\mathrm{SH}$ pulse contains three components: two of them at frequency $\omega_{20}=2 \omega_{10}$ but propagating with different velocities [described by Eq. (25)], and a third one [see Eq. (24)] at carrier frequency $2 \omega_{10}+\Omega_{0}$ propagating in between the first two [see Fig. 2(b)]. Note that, in the first-order dispersion approximation, the wave number for the fundamental and SH waves are, respectively, $k_{1}\left(\omega_{10}+\frac{\Omega}{2}\right)=$ $k_{10}+\frac{\Omega}{2 u_{1}}$ and $k_{2}\left(2 \omega_{10}+\Omega\right)=k_{20}+\frac{\Omega}{u_{2}}$. The phase mismatch at frequency $\Omega$ is defined as $\Delta k(\Omega)=k_{2}(2 \omega+\Omega)-2 k_{1}(\omega+$ $\left.\frac{\Omega}{2}\right)=\Delta k+v_{21} \Omega$. Therefore, the parameter $D(\Omega)$ may be interpreted as the phase mismatch: $\Delta k(\Omega) \equiv D(\Omega)$ clearly showing that the most efficient SHG occurs when $D(\Omega)=0$.
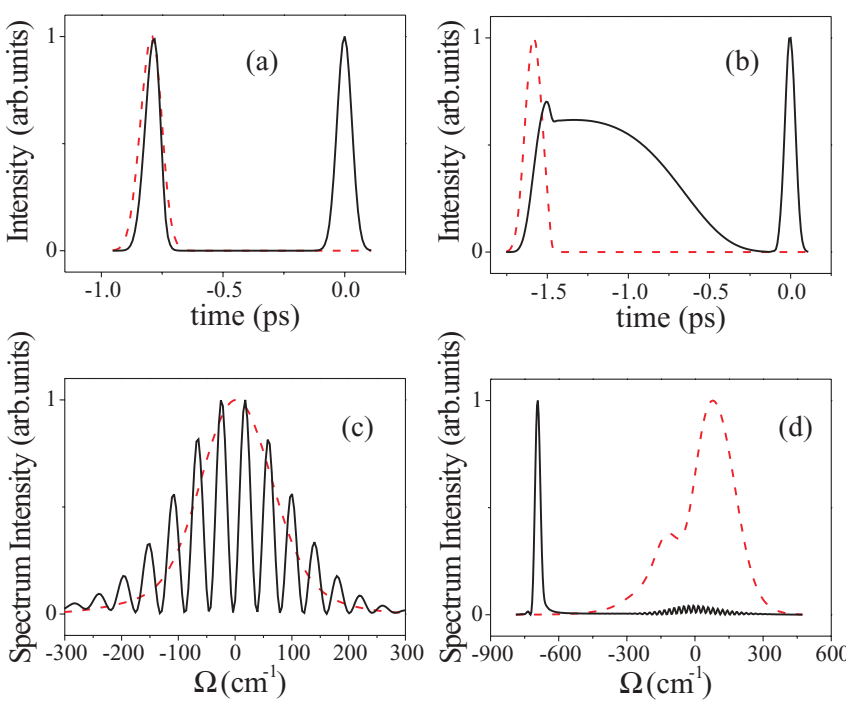

FIG. 2. (Color online) Numeric demonstration of Case $\mathrm{C}$ when the FH spectra function $F(\Omega)$ (a), (c) does not contain and (b), (d) does contain the frequency $\Omega_{0}$. The SH (solid line) and FH (dashed line) (a), (b) intensity and (c), (d) spectra profiles at the exit of the nonlinear medium are presented. The parameters (see text) used are (a), (c) $L=20 \mathrm{~mm}$; (b), (d) $L=40 \mathrm{~mm}$; (a), (c) $L_{v}=25 \mathrm{~mm}$, $L_{n}=1 \mathrm{~mm}, \tau=0.1 \mathrm{ps}, I_{\mathrm{FH}}=1 \mathrm{GW} / \mathrm{cm}^{2}, \Delta k=100 \mathrm{~cm}^{-1}$; and (b), (d) $\Delta k=50 \mathrm{~cm}^{-1}$, (b), (d).
The numerical example of the solution of Eq. (1) in the regime studied here (Case $C$ ) is presented in Fig. 2. The same $\{L=20 \mathrm{~mm}$ [Figs. 2(a) and 2(c)] and $L=40 \mathrm{~mm}$ [Figs. 2(b) and 2(d)]\} medium with switched-off GVD $\left(L g_{j}=\infty\right)$ and $L_{v}=25 \mathrm{~mm}, L_{n}=1 \mathrm{~mm}$ was used. The

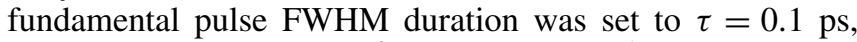
intensity $I_{\mathrm{FH}}=1 \mathrm{GW} / \mathrm{cm}^{2}, \Delta k=100 \mathrm{~cm}^{-1}$ [Figs. 2(a) and 2(c)] and $\Delta k=50 \mathrm{~cm}^{-1}$ [Figs. 2(b) and 2(d)].

Note that when the component at $\Omega_{0}$ in the $\mathrm{FH}$ wave is absent, the SH pulse (as predicted by the above theory) has a double-peak profile [Fig. 2(a)] composed by the free and the driven wave solutions [Eqs. (19) and (20)]. The spectrum [Fig. 2(c)] of the $\mathrm{SH}$ radiation has fringes as the result of the temporal delay between two spikes propagating with different velocities. We mention that the double-peaked profiles in phase-mismatched SHG under conditions of group-velocity mismatch were also studied by other authors [10,11,14]. However, if the component at $\Omega_{0}$ is present $\left[F\left(\Omega_{0}\right) \neq 0\right]$, the new component at shifted frequency appears [Fig. 2(d)]. The temporal duration of such a component is increasing with the propagation according to the solution given by Eq. (24) [Fig. 2(b)].

The cases of phase-mismatched SHG in the first-order dispersion approximation discussed so far are summarized in Fig. 3. The square FH pulse profile spectrum $F(\Omega)$ is presented in Fig. 3(a) for narrowband (dashed line) and broadband (solid line) FH pulse. The dispersion of the fundamental wave is shown in Fig. 3(b) (dashed line) by plotting $K_{1}(\Omega)=$ $2 k\left(\omega_{10}+\Omega / 2\right)$ as function of the frequency $\Omega$. The SH wave number $K_{2}(\Omega)=k\left(\omega_{20}+\Omega\right)$ as function of $\Omega$ is also shown for comparison (solid line). Note that the slopes of the plotted lines give the group velocities of the interacting pulses. In the plot, $v_{21}>0\left(u_{2}<u_{1}\right)$. The nonzero phase mismatch at carrier frequency $(\Omega=0)$ is persistent [in the presented case $\Delta k>0$, see Fig. 3(b)]. We can see that, because of the different slopes, the exact phase matching occurs at a shifted frequency $\Omega_{0}=-\frac{\Delta k}{v_{21}}$ [intersection point in Fig. 3(b)]. Thus, if the bandwidth of the fundamental wave is narrow [Fig. 3(a), dashed line], the SH pulse contains two components around $\Omega=0$ : the component [denoted by (1) in the figure] propagating with the velocity of the $\mathrm{SH}$ in linear medium $\left(u_{2}\right)$ and corresponding to the free $\mathrm{SH}$ wave solution, and the driven wave component [denoted by (2)] propagating with the group velocity $u_{1}$ of the polarization source (equal to that of the FH). Due to their different velocities, these two components possess a relative delay proportional to the distance, i.e., $\propto\left|v_{21} z\right|$ [see Fig. 3(d)], and lead to interference fringes in the $\mathrm{SH}$ spectrum close to $\Omega=0$ [see Fig. 3(c)]. However, in the case of broadband FH [Fig. 3(a), solid line], the nonzero component at frequency $\omega_{10}+\Omega_{0} / 2, F\left(\Omega_{0}\right) \neq 0$ generates a phase-matched SH component $\left[\Delta k\left(\Omega_{0}\right)=D\left(\Omega_{0}\right)=0\right]$ in proximity to the frequency $\omega_{20}+\Omega_{0}$ [see in Fig. 3(c) the component denoted by (3)]. The spectral bandwidth of the phase-matched component (3) shrinks because of the filtering described by the function $\operatorname{sinc}\left(\frac{\nu_{21}\left(\Omega-\Omega_{0}\right) z}{2}\right)$ [see Eq. (24)]. Note that the spectral amplitude of the component (3) is growing proportional to $z$. In the temporal domain, its dynamics corresponds to the formation of a long pulse with duration increasing proportionally to $z$ [see component (3) in Fig. 3(d)]. 

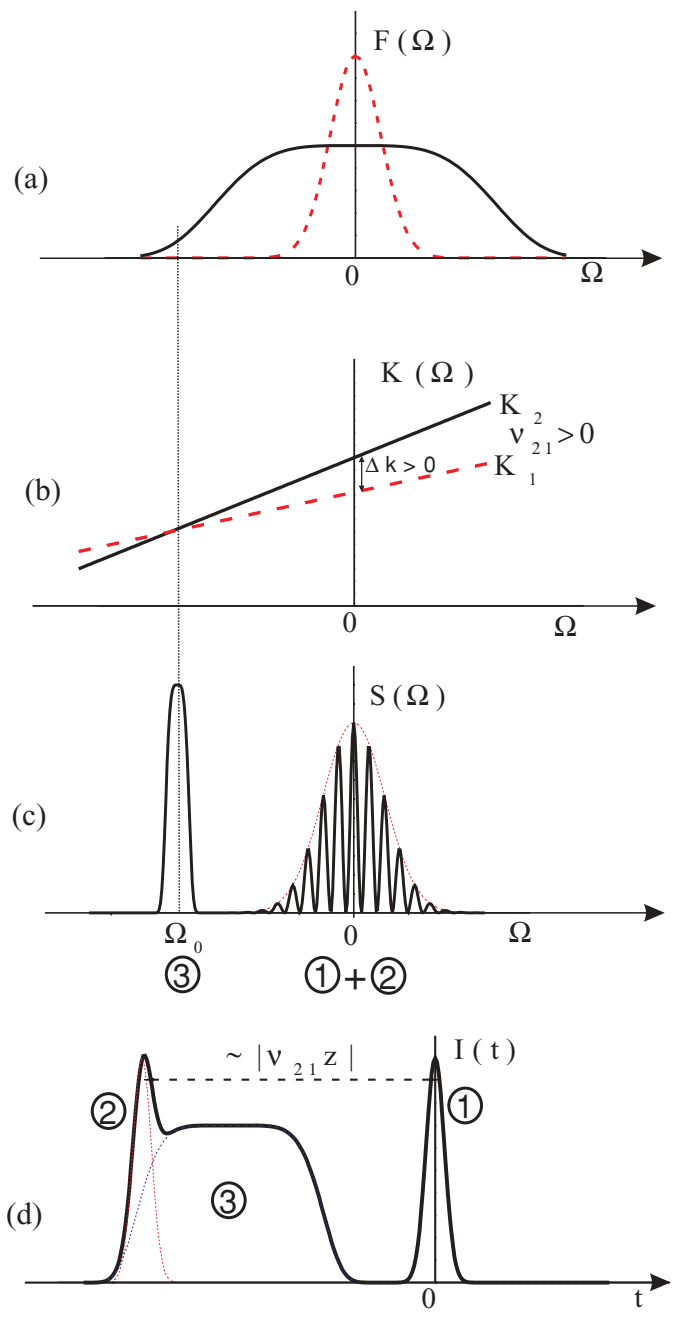

FIG. 3. (Color online) Illustration and explanation of the different $\mathrm{SH}$ components in phase-mismatched SHG. The GVM dominant case.

Case D: Regime of large group-velocity dispersion (GVD dominating over GVM). As mentioned before, the quantity $D(\Omega)$ may be interpreted as the phase mismatch at the detuned frequency $\Omega$. Indeed, the $\mathrm{SH}$ wave number within the secondorder approximation is $k_{2}\left(\omega_{20}+\Omega\right)=k_{20}+\frac{\Omega}{u_{2}}+\frac{1}{2} g_{2} \Omega^{2}$. On the other hand, according to our model, the $\mathrm{FH}$ wave number accounts just for the first-order dispersion (the reference frame is moving with the fundamental group velocity $u_{1}$ ), therefore, $k_{1}\left(\omega_{10}+\frac{\Omega}{2}\right)=k_{10}+\frac{\Omega}{2 u_{1}}$. The phase mismatch can thus be rewritten as $\Delta k(\Omega)=k_{2}\left(2 \omega_{10}+\Omega\right)-2 k_{1}\left(\omega_{10}+\frac{\Omega}{2}\right)=\Delta k+$ $v_{21} \Omega+\frac{1}{2} g_{2} \Omega^{2}=D(\Omega)$. Obviously, efficient SH generation occurs when $D(\Omega)=0$ (perfect phase matching). We can see how this condition can be implemented by rewriting $D(\Omega)$ [introduced by Eq. (7)] as

$$
D(\Omega)=D_{0}+\left(\nu_{21}+g_{2} \Omega_{0}\right)\left(\Omega-\Omega_{0}\right)+\frac{1}{2} g_{2}\left(\Omega-\Omega_{0}\right)^{2},
$$

where

$$
D_{0}=\Delta k+v_{21} \Omega_{0}+\frac{1}{2} g_{2} \Omega_{0}^{2} .
$$

Because of material dispersion, the condition $D(\Omega)=0$ can not be guaranteed for all frequency ranges, but only for some fixed frequency $\Omega_{0}$, where $D_{0}=D\left(\Omega_{0}\right)=0$. In the case of dispersion limited to the first order, this gives $\Omega_{0}=-\frac{\Delta k}{\nu_{21}}$ and the resulting dynamics is the one discussed in the preceding Case C. In the case of significant GVD, the phase matching occurs for the two frequencies given by

$$
\Omega_{0}^{(1),(2)}=-\frac{\nu_{21}}{g_{2}} \pm \frac{\nu_{21}}{g_{2}} \sqrt{1-\frac{2 \Delta k g_{2}}{v_{21}^{2}}},
$$

while in the case of moderate GVD $\left(\frac{2 \Delta k g_{2}}{v_{21}^{2}} \ll 1\right)$, these are

$$
\begin{gathered}
\Omega_{0}^{(1)} \approx-\frac{\Delta k}{v_{21}}, \\
\Omega_{0}^{(2)} \approx \frac{\Delta k}{v_{21}}-\frac{2 v_{21}}{g_{2}} .
\end{gathered}
$$

In the case of group-velocity matching $\left(v_{21}=0\right)$, the frequency detuning due to self-phase matching is characterized by the two symmetrical frequency values

$$
\Omega_{0}^{(1),(2)}= \pm \sqrt{-\frac{2 \Delta k}{g_{2}}} .
$$

The appearance of the phase-matched components in the case of large GVD is depicted in Fig. 4. For this purpose, we introduce the function $K_{2}(\Omega)=k_{2}\left(\omega_{20}+\Omega\right)-\Omega / u_{1}=$ $k_{20}+\frac{1}{2} g_{2}\left(\Omega+v_{21} / g_{2}\right)^{2}-v_{21}^{2} / 2 g_{2}$ (as a SH wave number in the coordinate frame moving with $u_{1}$ velocity) and we rewrite parameter $D$ as $D(\Omega)=\left[k_{2}\left(\omega_{20}+\Omega\right)-\Omega / u_{1}\right]-$ $2\left[k_{1}\left(\omega_{10}+\Omega / 2\right)-\Omega / 2 u_{1}\right]$. Also, note that, in our approximation, $k_{1}\left(\omega_{10}+\Omega / 2\right)-\Omega / 2 u_{1}=k_{10}$, so we obtain $D(\Omega)=$ $K_{2}(\Omega)-2 k_{10}$. Therefore, the phase-matched components in Fig. 4 [graphic solution of the equation $D(\Omega)=0$ ] occur at the intersection of the curve $K_{2}=K_{2}(\Omega)$ with the straight horizontal line $K_{1}=2 k_{10}$. If group-velocity matching takes place $\left(v_{21}=0\right)$, the phase-matched components are displaced symmetrically [Figs. 4(a) and 4(b)]. Intersection takes place only if $\Delta k g_{2}<0$ [see Eq. (30) and Figs. 4(a) and 4(b)]. The
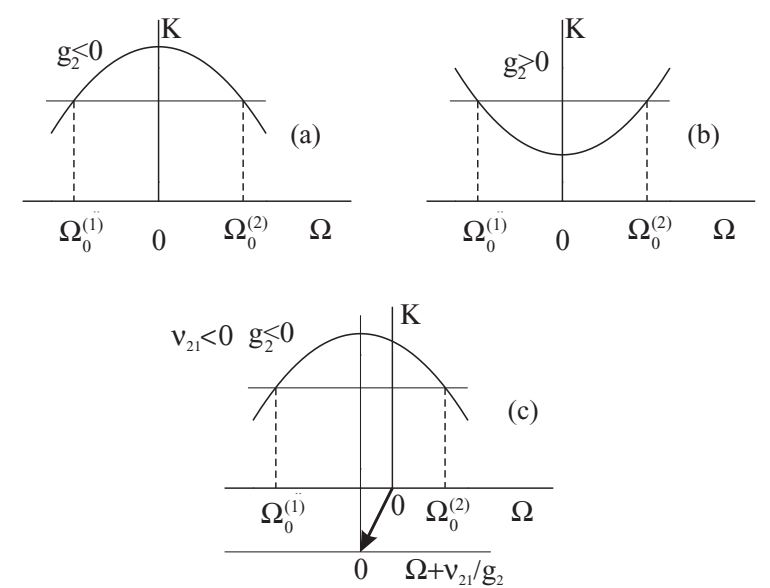

FIG. 4. Appearance of the phase-matched components in phasemismatched SHG with the GVD dominant over GVM. (a), (c) $\Delta k>0$ and (b) $\Delta k<0$. 

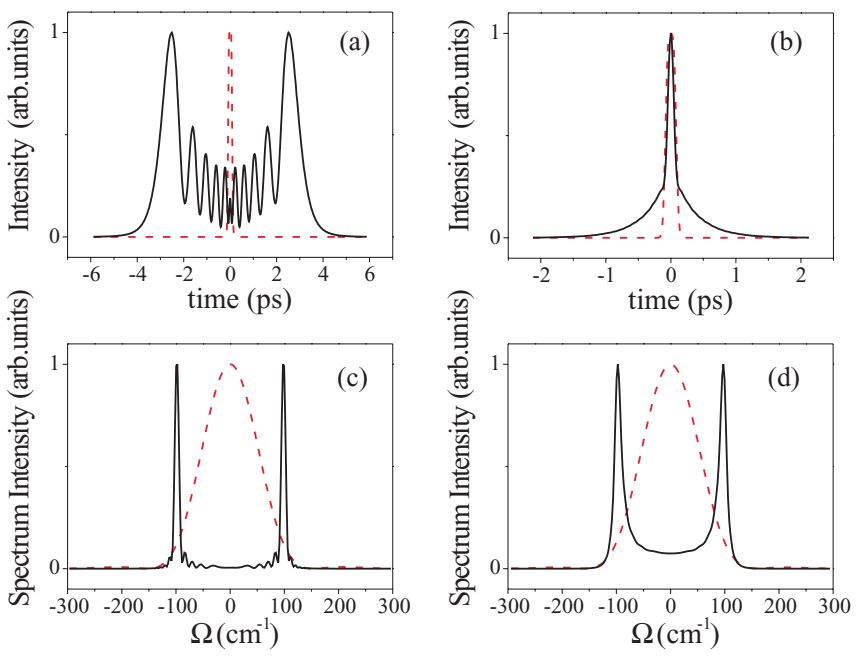

FIG. 5. (Color online) Numeric demonstration of Case D (GVD dominant over GVM). The SH (solid line) and FH (dashed line) (a), (b) intensity and (c), (d) spectra profiles at the exit of the nonlinear medium are presented. The left column [(a), (c)] depicts the case without linear losses and the right column [(b), (d)] depicts the case with linear losses. The parameters (see text) used are $L=10 \mathrm{~mm}$, $L_{g 2}=20 \mathrm{~mm}, L_{n}=1 \mathrm{~mm}, \tau=0.1 \mathrm{ps}, I_{\mathrm{FH}}=1 \mathrm{GW} / \mathrm{cm}^{2}$, and $\Delta k=$ $-30 \mathrm{~cm}^{-1}$. GVM matched interaction $\left(L_{v}=\infty\right)$. For the dissipative case [(b), (d)], $L_{\alpha}=1 \mathrm{~mm}$.

presence of GVM shifts the parabola $K_{2}(\Omega)$, respectively, to the left by a frequency amount of $\nu_{21} / g_{2}$ and down by an amount $\Delta k=v_{21}^{2} / 2 g_{2}$ [see Fig. 4(c)].

The numerical illustration of the Case $\mathrm{D}$ in the regime of perfect group-velocity matching is shown in Fig. 5. The $L=10 \mathrm{~mm}$ crystal with GVD $\left(L g_{2}=20 \mathrm{~mm}\right)$ and $L_{n}=$ $1 \mathrm{~mm}$ was used. The fundamental pulse FWHM duration was set to $\tau=0.1 \mathrm{ps}$, intensity $I_{\mathrm{FH}}=1 \mathrm{GW} / \mathrm{cm}^{2}$, and $\Delta k=$ $-30 \mathrm{~cm}^{-1}$. When absorption is negligible, the short FH pulse [see Fig. 5(a), dashed line] generates broad SH pulse [Fig. 5(a), solid line) with the temporal profile exhibiting modulation caused by interference of two spectral components [Fig. 5(c), solid line]. The same case with the linear absorbtion $\left(L_{\alpha}=\right.$ $2 \mathrm{~mm}$ ) is presented in Figs. 5(b) and 5(d), where only the driven $\mathrm{SH}$ wave survives and sustains the short $\mathrm{SH}$ pulse formation.

\section{B. Three-dimensional SHG}

In the following treatment, we remove the plane-wave approximation, supposing that the $\mathrm{FH}$ beam is limited in transverse space. We shall investigate the SHG process considering cylindrically symmetric beams. In this case, we assume $B_{j}=B_{j}(t, r), r=\sqrt{x^{2}+y^{2}}$, and, for the transverse Laplace operator, $\Delta_{\perp}=\partial^{2} / \partial r^{2}+(1 / r) \partial / \partial r$. In such a case, instead of Eq. (3), we have

$\frac{\partial B_{2}}{\partial z}+v_{21} \frac{\partial B_{2}}{\partial t}-\frac{i}{2} g_{2} \frac{\partial^{2} B_{2}}{\partial t^{2}}+\frac{i}{2 k_{20}} \Delta_{\perp} B_{2}+i \Delta k B_{2}=\sigma_{2} B_{1}^{2}$.
We look for the solution of Eq. (31) in the spectral domain by using the Fourier-Hankel transform. We have

$$
\begin{gathered}
B_{2}=\int_{0}^{\infty} \int_{-\infty}^{\infty} S(\Omega, \kappa, z) J_{0}(\kappa r) \exp (i \Omega t) \frac{d \Omega}{2 \pi} \kappa d \kappa, \\
B_{1}^{2}=\int_{0}^{\infty} \int_{-\infty}^{\infty} F(\Omega, \kappa) J_{0}(\kappa r) \exp (i \Omega t) \frac{d \Omega}{2 \pi} \kappa d \kappa .
\end{gathered}
$$

The substitution of Eq. (32) into (31) and the Fourier-Hankel transform give the same equation [Eq. (6)] as in the 1D case

$$
\frac{\partial S(\Omega, \kappa, z)}{\partial z}+i D(\Omega, \kappa) S(\Omega, \kappa)=\sigma_{2} F(\Omega, \kappa),
$$

where

$$
D(\Omega, \kappa)=\Delta k+v_{21} \Omega+\frac{1}{2} g_{2} \Omega^{2}-\frac{\kappa^{2}}{2 k_{20}} .
$$

The solution of Eq. (33) is given by Eq. (8), but now the functions $F$ and $D$ depend also on the spatial frequency $\kappa$ :

$$
S=z \sigma_{2} F(\Omega, \kappa) \operatorname{sinc}\left(\frac{D(\Omega, \kappa) z}{2}\right) \exp \left(-i \frac{D(\Omega, \kappa) z}{2}\right) \text {. }
$$

As discussed previously, the most effective SH generation ( $S$ growing proportionally to $z$ ) takes place when $D=0$. In the $1 \mathrm{D}$ case, this condition may be satisfied only for fixed frequency(ies) $\Omega_{0}$. In contrast, in $3 \mathrm{D}$, the dependence of $D$ on the spatial frequency $\kappa$ leads to extra degrees of freedom, and $D=0$ may be satisfied within a whole frequency range over which the $\mathrm{SH}$ wave has a suitable angular dispersion (dependence of the transverse wave vector on generated frequency). In other words, we have $\kappa=\kappa(\Omega)$ and $D[\Omega, \kappa(\Omega)]=0$, leading from Eq. (34) to

$$
\frac{\kappa^{2}(\Omega)}{2 k_{20}}=\Delta k+v_{21} \Omega+\frac{1}{2} g_{2} \Omega^{2} .
$$

Note that the spatial frequency describing the angular dispersion $\kappa=\kappa(\Omega)$ is by definition the transverse wave vector $k_{2 \perp}=k_{2} \sin (\theta) \equiv \kappa(\Omega)$.

As shown in the $1 \mathrm{D}$ case, here also $D$ may be interpreted as the longitudinal phase mismatch. This can be proved by rewriting the longitudinal $\mathrm{SH}$ wave-vector component as

$$
k_{2 z}(\omega)=\sqrt{k_{2}^{2}(\omega)-\kappa^{2}} \approx k_{2}(\omega)-\frac{\kappa^{2}}{2 k_{20}} .
$$

Considering that $\omega$ is the absolute frequency $\left(\omega=\omega_{20}+\Omega\right)$, by expanding the first term up to second order $k_{2}\left(\omega_{20}+\Omega\right)=$ $k_{20}+\frac{\Omega}{u_{2}}+\frac{1}{2} g_{2} \Omega^{2}$, we obtain

$$
k_{2 z}\left(\omega_{20}+\Omega\right) \approx k_{20}+\frac{\Omega}{u_{2}}+\frac{1}{2} g_{2} \Omega^{2}-\frac{\kappa^{2}}{2 k_{20}} .
$$

In order to interpret $D$ as the longitudinal phase mismatch, we rewrite the fundamental wave vector at frequency $\omega_{10}+$ $\Omega / 2$ as

$$
k_{1 z}\left(\omega_{10}+\Omega / 2\right)=k_{10}+\frac{\Omega}{2 u_{1}},
$$

corresponding to undepleted (invariant during propagation) fundamental harmonic approximation. Thus, the longitudinal phase mismatch defined as $\Delta k_{z}(\Omega)=k_{2 z}\left(\omega_{20}+\Omega\right)-2$ 
$k_{1 z}\left(\omega_{10}+\Omega / 2\right)$ becomes equal to the parameter $D$ defined by Eq. (34): $\Delta k_{z}(\Omega)=D(\Omega, \kappa)$. We can say that the optimal SH generation condition $D=0$ [defined by Eq. (36)], being guaranteed by the angular dispersion of the generated $\mathrm{SH}$, corresponds to longitudinal phase matching between the angularly dispersed SH components and the frequency components of the fundamental wave. Note that the phase-matching process is selecting from the bulk FH spectrum $(\Omega, \kappa$ volume) the components described by Eq. (36).

We see from Eq. (34) that $D(\Omega, \kappa)$ calculated at $\kappa=0$ (axial component) is equivalent to the parameter $D(\Omega)$ in the $1 \mathrm{D}$ case, $D(\Omega, 0)=D(\Omega)$. Therefore, on-axis phase matching in the three-dimensional case can be reached at the same shifted frequency $\Omega_{0}$ found in the one-dimensional case. The condition described by Eq. (36) ensuring optimal SHG can be rewritten by introducing the frequency shift $\Omega_{0}$ defined via $D_{0}=D\left(\Omega_{0}\right)=0$ [see Eq. (27)]:

$$
\frac{\kappa^{2}(\Omega)}{2 k_{20}}=\left(\nu_{21}+g_{2} \Omega_{0}\right)\left(\Omega-\Omega_{0}\right)+\frac{1}{2} g_{2}\left(\Omega-\Omega_{0}\right)^{2} .
$$

While $D=0$ is satisfied for the on-axis spectral component at the shifted frequency $\left[\Omega_{0}, k_{2 \perp}=\kappa\left(\Omega_{0}\right)=0\right]$, for the off-axis components $\left[k_{2 \perp}=\kappa(\Omega) \neq 0\right]$, the optimal SHG condition occurs for frequencies $\Omega \neq \Omega_{0}$, satisfying the above equation (40).

It is interesting to note that the occurrence of longitudinal phase matching, guaranteed by the frequency shift on axis, and the angular dispersion indicate the reshaping of the generated SH pulse into an X pulse [36]. We can show this by substituting Eq. (36) into (38), and obtaining $k_{2 z}\left(\omega_{20}+\Omega\right)=k_{20}-\Delta k+$ $\frac{\Omega}{u_{1}}$. Taking into account that $-\Delta k=v_{21} \Omega_{0}+\frac{1}{2} g_{2} \Omega_{0}^{2}$ and $k_{2}\left(\omega_{20}+\Omega_{0}\right)=k_{20}+\frac{\Omega_{0}}{u_{2}}+\frac{1}{2} g_{2} \Omega_{0}^{2}$, we have

$$
k_{2 z}\left(\omega_{20}+\Omega\right)=k_{2}\left(\omega_{20}+\Omega_{0}\right)+\frac{\Omega-\Omega_{0}}{u_{1}} .
$$

Considering the absolute frequencies $\omega=\omega_{20}+\Omega$, and introducing a new carrier frequency $\omega_{20}^{(\text {new) }}=\omega_{20}+\Omega_{0}$, Eq. (41) can be written as

$$
k_{2 z}(\omega)=k_{2}\left(\omega_{20}^{(\text {new })}\right)+\frac{\omega-\omega_{20}^{(\text {new })}}{u_{1}} .
$$

Note that the longitudinal wave vector does not depend on the spatial frequency but is linearly proportional to the temporal frequency. Such dependence sustains the wave-packet invariance during propagation [36] and X-wave formation. We mention that the analytic solution showing possible $\mathrm{SH}$ $\mathrm{X}$-pulse formation in the undepleted regime and first-order dispersion was obtained also in [34].

In summary, from this analysis, we have found that the optimal SH generation condition $D(\Omega, \kappa)=0$, also equivalent to the longitudinal phase matching $\Delta k_{z}(\Omega)=0$, is satisfied for on-axis component(s) at shifted frequency(ies) $\omega_{20}^{\text {(new) }}=\omega_{20}+$ $\Omega_{0}\left[\Omega_{0}\right.$ defined by Eqs. (28)-(30)], as well as for angularly dispersed off-axis components as shown by Eq. (42). This corresponds to a dispersion of the $\mathrm{SH}$ wave-vector longitudinal component described by Eq. (42), which meets the definition of the X-pulse propagating with the group velocity $u: k_{z}(\omega)=$ $\gamma+\frac{\omega}{u}(\gamma=$ const $)[36]$.

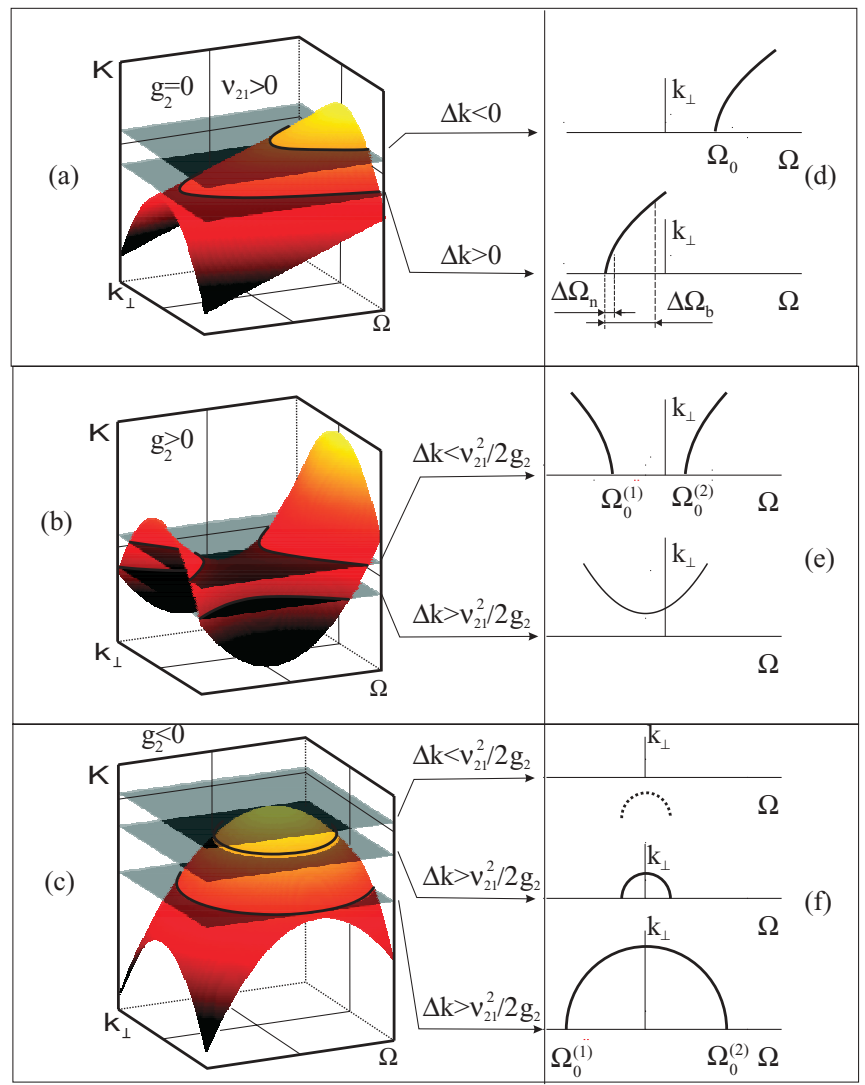

FIG. 6. (Color online) The phase-matching (left column) and angular dispersion features (right column) in the 3D case. (a), (d) depict the GVM dominant case. The GVD dominant case is presented in (b), (e) for $g_{2}>0$ and in (c), (f) for $g_{2}<0$. The dotted line in (f) depicts a situation where the phase matching does not exist.

As mentioned above, the transverse wave-vector dependence on frequency $\left[k_{\perp}=\kappa(\Omega)\right]$ defines the angular dispersion and can be illustrated graphically as the longitudinal phase matching (Fig. 6). For this reason, we introduce $K_{2}(\Omega, \kappa)=$ $k_{20}+v_{21} \Omega+\frac{1}{2} g_{2} \Omega^{2}-\frac{\kappa^{2}}{2 k_{20}}$ as the SH longitudinal wave vector in the reference frame moving with the velocity $u_{1}$ (we have subtracted the term $\left.\frac{\Omega}{u_{1}}\right)$, thus, $\Delta k_{z}(\Omega)=D(\Omega, \kappa)=$ $K_{2}(\Omega, \kappa)-2 k_{10}$. The longitudinal phase-matching condition $D(\Omega, \kappa)=0$ graphically is equivalent to the intersection of the surface $K_{2}=K_{2}(\Omega, \kappa)$ with the plane $K_{1}=2 k_{10}$ (see Fig. 6).

In the following, we discuss different regimes.

Case 1. In the case of phase-mismatched SHG with GVM dominant over GVD $\left(\Delta k \neq 0, v_{21} \neq 0, g_{2} \cong 0\right)$, we have $D=$ 0 , when

$$
\frac{\kappa^{2}}{2 k_{20}}=\Delta k+v_{21} \Omega=\Delta k+v_{21} \Omega_{0}+v_{21}\left(\Omega-\Omega_{0}\right) .
$$

On-axis $(\kappa=0)$ longitudinal phase matching leads to the generation of the $\mathrm{SH}$ component at a frequency shifted by $\Omega_{0}=-\frac{\Delta k}{v_{21}}$ (as in the one-dimensional case), while off-axis phase matching occurs for transverse wave-vector components satisfying

$$
k_{\perp}(\Omega)=\sqrt{2 k_{20} \nu_{21}\left(\Omega-\Omega_{0}\right)} .
$$

The situation we have when $\nu_{21}>0$ is depicted in Figs. 6(a) and 6(d). If $v_{21}<0$, the figure is the same, but the 
axis of the temporal frequency $\Omega$ should be inverted. Note that the appearance of a single angular dispersion branch sustains the kind of X-wave formation in SH when $\Omega \geqslant \Omega_{0}$ if $\nu_{21}>0$ (and when $\Omega \leqslant \Omega_{0}$ if $\nu_{21}<0$ ).

Case 2. In the case of normal GVD $\left(g_{2}>0\right)$, the longitudinal phase matching may be described as the intersection of the saddle surface $K_{2}=K_{2}(\Omega, \kappa)$ and the horizontal plane $K_{1}=2 k_{10}$ [see Fig. 6(b)]. If $\Delta k<\frac{v_{21}^{2}}{2 g_{2}}$, the intersecting plane is above the saddle point and two branches of the angular dispersion appear, starting from two distinct axial frequencies $\Omega_{0}^{(1)}$ and $\Omega_{0}^{(2)}$ defined by Eqs. (28) and (29) [see Figs. 6(b) and 6(e)]. The off-axis components $\Omega<\Omega_{0}^{(1)}$ and $\Omega>\Omega_{0}^{(2)}$ (if $\Omega_{0}^{(1)}<\Omega_{0}^{(2)}$ ) also become phase matched when

$$
k_{\perp}(\Omega)=\sqrt{g_{2} k_{20}\left(\Omega-\Omega_{0}^{(1)}\right)\left(\Omega-\Omega_{0}^{(2)}\right)} .
$$

Therefore, the appearance of angular dispersion with the gap in temporal frequency (no components for $\Omega_{0}^{(1)}<\Omega<$ $\Omega_{0}^{(2)}$ ) sustains the subluminal X-wave formation in $\mathrm{SH}$ [see Fig. 6(e)].

If $\Delta k>\frac{v_{21}^{2}}{2 g_{2}}$, the intersecting plane is below the saddle point and on-axis phase matching becomes impossible (solutions for axial frequencies $\Omega_{0}^{(1),(2)}$ do not exist). However, off-axial components can be phase matched, as shown in Fig. 6(e) (bottom line). That sustains the formation of the superluminal X wave.

In the case of group-velocity matching $\left(v_{21}=0\right)$, the topology of the phase matching remains unchanged, but the picture becomes symmetric: the on-axis phase matching occurs when $\Delta k<0$ at frequencies

$$
\Omega_{0}^{(1),(2)}= \pm \sqrt{\frac{-2 \Delta k}{g_{2}}},
$$

and it occurs for off-axis frequency components satisfying

$$
k_{\perp}(\Omega)=\sqrt{g_{2} k_{20}\left(\Omega^{2}-\Omega_{0}^{2}\right)} .
$$

Case 3. In the case of anomalous GVD $\left(g_{2}<0\right)$, the longitudinal phase matching may be depicted as the intersection of the ellipsoid $K_{2}=K_{2}(\Omega, \kappa)$ and the plane $K_{1}=2 k_{10}$ [see Fig. 6(c)]. Intersection occurs if $\Delta k>\frac{v_{21}^{2}}{2 g_{2}}\left(\Delta k>0\right.$ if $\left.\nu_{21}=0\right)$. The same expressions for the axial components given by $\Omega_{0}^{(1),(2)}$ [Eqs. (28) and (46)] and for the transverse wave vectors [Eqs. (45) and (47)] are valid. The intersection curves are ellipses and the phase-matched frequency range lies in-between $\Omega_{0}^{(1)}$ and $\Omega_{0}^{(2)}$ [see Fig. 6(f)]. In this case, it is interesting to note that the appearance of angular dispersion sustains the formation of an $\mathrm{O}$ wave [36] in the $\mathrm{SH}$.

\section{CHANGE OF THE WAVE-PACKET TOPOLOGY VIA TRANSITION FROM 1D TO 3D INTERACTION}

In the following, we shall focus our attention on the regime of phase-mismatched SHG with GVM dominant over GVD (Case 1 of the preceding section), as this is the regime permitting us to observe in a spectacular way the transition from the one- to the three-dimensional dynamics of the SHG process. It is worth noting that a broad FH beam, and consequently a narrow angular spectrum, allow us to access (thanks to the self-phase-matching process studied above) only the narrow spectral range $\Delta \Omega_{n}$ of the generated temporal bandwidth, starting from frequency $\Omega_{0}$ [see Fig. 6(d)], and therefore support the formation of a long SH pulse [denoted as component (3) as discussed in Sec. II A] in the 1D Case C.

On the contrary, a narrow $\mathrm{FH}$ beam possessing a broad angular spectrum allows us to access a broad temporal bandwidth $\Delta \Omega_{b}$ featured also by angular dispersion [see Fig. 6(d)]. This type of spectrum sustains the formation of a short X-type SH pulse characterized by a central spike duration proportional to the whole spectrum bandwidth $\sim \Delta \Omega_{b}$.

The above considerations suggest that a decrease of the FH beam diameter should cause a transition from a long axial $\mathrm{SH}$ pulse toward a short $\mathrm{X}$ pulse. We have demonstrated this effect in a numerical experiment of phase-mismatched SHG under conditions of large GVM. We evaluated the numerical solution of Eq. (1) for different input conditions (diameters of the fundamental beam and phase mismatch). The $L=40 \mathrm{~mm}$ medium with switched-off GVD and $L_{v}=25 \mathrm{~mm}, L_{n}=1$ $\mathrm{mm}, \Delta k=-50 \mathrm{~cm}^{-1}$ was used. The diffraction was accounted for by means of the diffraction lengths (Rayleigh ranges) $L_{d j}=k_{j 0} d_{0}^{2} /(4 \ln 2)$, calculated for $d_{0}=1 \mathrm{~mm}$ diameter beam at FWHM. It was set at $L_{d 1}=3 \mathrm{~m}$ for $\mathrm{FH}$ and $L_{d 2}=$ $6 \mathrm{~m}$ for SH. The fundamental pulse duration was $\tau=0.1 \mathrm{ps}$, and the intensity $I_{\mathrm{FH}}=1 \mathrm{GW} / \mathrm{cm}^{2}$. Results of the numerical simulation are presented in Fig. 7.

First, we started with a fundamental wave with a narrow angular spectrum (FH diameter $d=1 \mathrm{~mm}$ ). The temporal [Fig. 7(a)] and spectral [Fig. 7(b)] profiles of the SH radiation generated at the very beginning $(z=0.07 \mathrm{~mm})$ of the nonlinear crystal resemble the temporal profile of the $\mathrm{FH}$ and the corresponding spectrum [defined by function $F(\Omega)$ ]. After $z=40 \mathrm{~mm}$ of propagation, the SH wave [see Figs. 7(c) and 7(d)] develops features typical of the 1D dynamics: the free $\mathrm{SH}$ component [see, for comparison, the 1D Case C component (1) in Fig. 3] generated at frequency $\omega_{20}$ propagates with the group velocity $u_{2}$; however, the driven $\mathrm{SH}$ component [as component (2) in Fig. 3], also generated at frequency $\omega_{20}$, propagates with the group velocity of the fundamental wave $\left(u_{1}\right)$. The interference of these temporally delayed components generates fringes around the frequency $\omega_{20}$ [see Fig. 7(d)]. However, the SH component generated at the shifted frequency $\omega_{20}+\Omega_{0}$ [Fig. 7(d)] [like the component (3) in Fig. 3)] is a broad pulse component with a nearly rectangular profile. Note here that $\Delta k<0, v_{21}>0$, thus, the frequency shift is positive $\left(\Omega_{0}>0\right)$. The $\mathrm{SH}$ pulse duration increases continuously [Fig. 7(c)], while the temporal spectrum shrinks until it reaches a distribution free from angular dispersion [Fig. 7(d)] (spectral distribution pattern lies along a line orthogonal to the $\omega$ axis). Note the total SH pulse profile on axis [inset of Fig. 7(c)] is identical to the profile obtained in the $1 \mathrm{D}$ calculation [see Fig. 2(b)].

Second, seeking to demonstrate the transition from the $1 \mathrm{D}$ to the $3 \mathrm{D}$ dynamics, we repeated the calculation with a 20 times smaller $\mathrm{FH}$ beam diameter $(d=50 \mu \mathrm{m})$. In order to see the FH beam diameter (and angular bandwidth) pure influence on the SHG dynamics, we switched off the diffraction of the fundamental beam $\left(L_{d 1}=\infty, L_{d 2}=6 \mathrm{~m}\right)$. The SH component at shifted frequency $\omega_{20}+\Omega_{0}$ [Fig. 7(f)] 

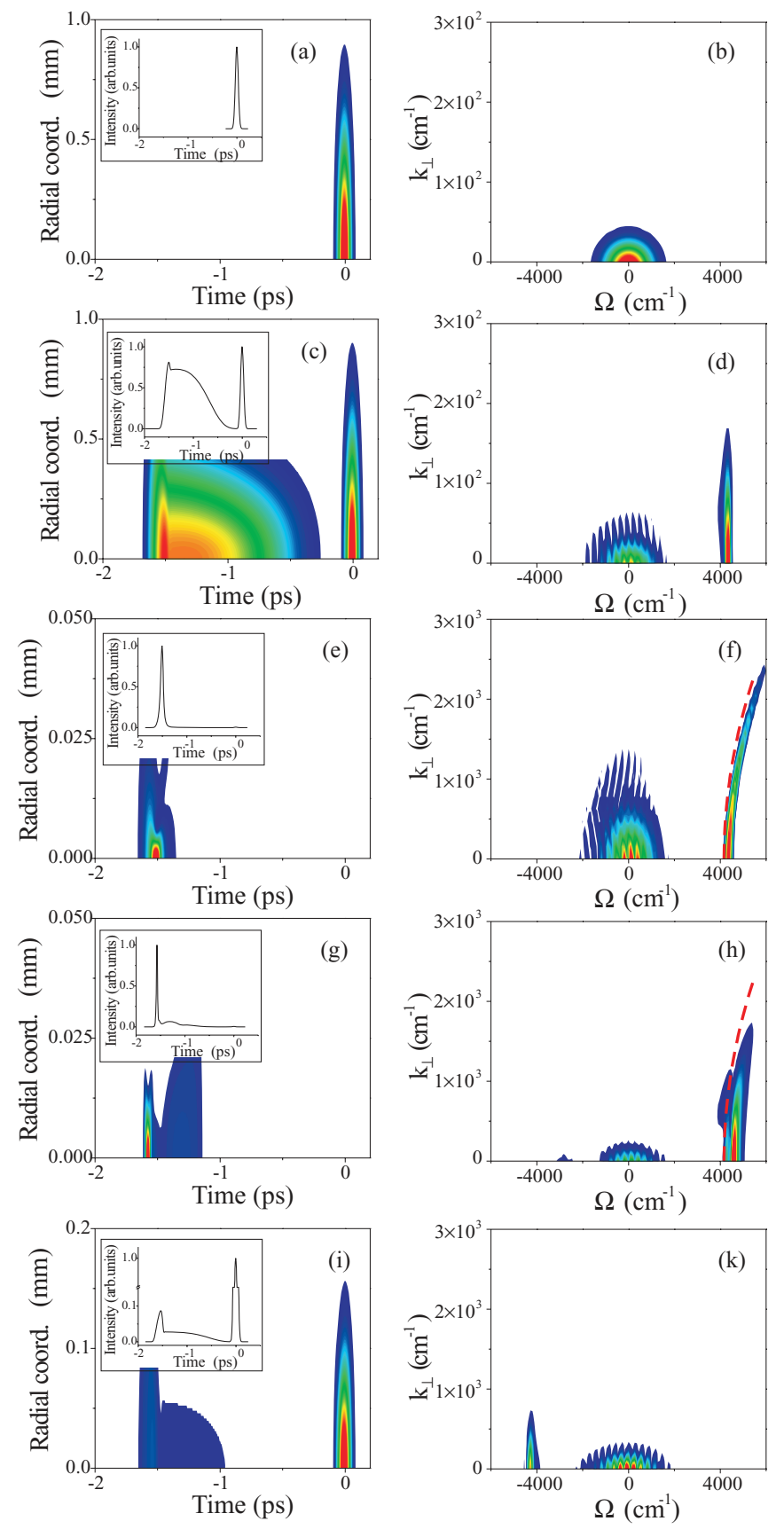

FIG. 7. (Color online) SH spatiotemporal profiles (left column) and corresponding spectrum intensity distributions (right column) for different phase-mismatched SHG cases obtained by numerical solution of the propagation equations. See text for details.

becomes bent, i.e., contains angular dispersion. The angular dispersion curves obtained analytically [Eq. (44)] are plotted in dashed lines. We observe a quite good agreement between theory and numerics. In contrast to the large beam case (when a long $\mathrm{SH}$ pulse is generated), here a narrow $\mathrm{FH}$ beam sustains a short X-pulse [Fig. 7(e), see also inset] formation with nearly constant duration. Note the tails typical of an X pulse.

Finally, the fact that the beam diffraction of the fundamental beam was switched off in the numerics is important for the experimental demonstration of the transition between 1D and 3D dynamics. Indeed, in reality, the diffraction of the
FH beam should be accounted for. However, the positive phase mismatch $(\Delta k>0)$ corresponds to a self-defocusing interaction case (due to effective Kerr nonlinearity induced by cascaded $\chi^{(2)}$ processes $[37,38]$ ), where the fundamental wave beam diameter is rapidly growing. On the contrary, the negative phase-mismatch $(\Delta k<0)$ case corresponds to a self-focusing dynamics for the fundamental beam and allows us to keep a narrow fundamental beam waist in the crystal. These conditions imply the natural possibility to demonstrate the transition from the $1 \mathrm{D}$ to the $3 \mathrm{D}$ case by changing $\Delta k$ via the crystal temperature tuning and, thus, by tuning the phase mismatch from positive to negative. We repeated the calculations for the self-focusing interaction $\left(\Delta k=-50 \mathrm{~cm}^{-1}\right)$ with the presence of diffraction $\left(L_{d 1}=3 \mathrm{~m}, L_{d 2}=6 \mathrm{~m}\right)$ and an initial FH beam diameter of $d=160 \mu \mathrm{m}$. During the interaction, the $\mathrm{FH}$ diameter shrinks and becomes narrower. Therefore, the SHG dynamics [Figs. 7(g) and 7(h)] is similar to the previous case with the narrow diameter $\mathrm{FH}$ and no diffraction. The component at shifted frequency [Fig. 7(h)] also presents angular dispersion, approximately matching the theoretical (dashed) line given by Eq. (44). The features of a short SH X pulse with weak tails emerge in Fig. 7(g). In the case of self-defocusing interaction $\left(\Delta k=50 \mathrm{~cm}^{-1}\right)$, with the other parameters set as above, the red-shifted frequency component appears [Fig. 7(k)]. However, the lack of angular dispersion determines a completely different pulse temporal dynamics [Fig. 7(i)] with features similar to the 1D case. The long SH component appears (see inset) as in the previously discussed case with a FH beam diameter of $d=1 \mathrm{~mm}$.

\section{EXPERIMENTAL RESULTS}

A set of experiments in different conditions was performed in the femtosecond-pulsed regime in order to illustrate, in phase-mismatched SHG, the dimensionality transition and the effect of reshaping of the interacting pulses. The experimental layout used for the measurements is illustrated in Fig. 8.

A traveling wave optical parametric amplifier (TOPAS) was pumped by a 130-fs, 800-nm pulse of a Ti:sapphire laser, generating signal and idler pulses at 1283 and $2124 \mathrm{~nm}$ wavelength, respectively. A 5-cm long lithium triborate crystal placed in an oven was pumped for SHG by the second harmonic of the idler, i.e., at $1062 \mathrm{~nm}$. The polarization of the latter (originally) vertical was changed into horizontal by means of a half-wave plate. By changing the distance $d$ between the lenses of an unbalanced telescope, we were able to control the beam size of the pump entering the nonlinear crystal, and thus its focusing conditions. The phase

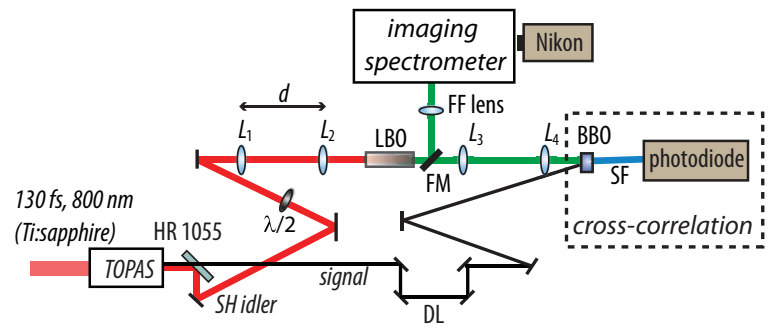

FIG. 8. (Color online) Scheme of the experimental layout used for the measurements of phase-mismatched second-harmonic generation. 
mismatch $\Delta k$ was controlled by tuning the crystal temperature. The diagnostics consisted of the detection of the far-field spatiotemporal spectrum $(\theta, \lambda)$ of the second-harmonic beam generated at the output of the LBO crystal by means of an imaging spectrometer combined with a NIKON camera. In addition, by flipping the mirror FM, the output radiation could be sent to a cross-correlation setup for the measurement of its temporal profile. In this case, the horizontally polarized TOPAS signal at $1283 \mathrm{~nm}$ was used as gate pulse for the sum frequency generation process occurring in a $300-\mu \mathrm{m}$ beta barium borate. Measurements of the $(\theta, \lambda)$ spectrum and the pulse temporal shape were performed for different values of the 1062-nm fundamental beam size, of the energy, and for different values of $\Delta k$, both in the self-defocusing and in the self-focusing regimes. In the following, we present experimental results that illustrate the $1 \mathrm{D} \leftrightarrow 3 \mathrm{D}$ transition of the phase-mismatched second-harmonic process, confirming that it can be observed as described in the preceding section by switching from the defocusing to the focusing regime.

\section{A. SHG in self-defocusing regime}

We start by considering the positive phase-mismatched case $(\Delta k>0)$ corresponding to a self-defocusing regime for the fundamental pump beam. In this case, the crystal temperature was tuned in order to be smaller than the exact phase-matching temperature. Figure 9 shows the spectra of the second-harmonic radiation generated by the LBO crystal when pumped by a fundamental pump beam of about $205 \mu \mathrm{m}$ FWHM, in two different cases. In Fig. 9(a), the spectrum corresponds to $\Delta k=23.1 \mathrm{~cm}^{-1}$ (top figure) and in Fig. 9(b) to $\Delta k=55.2 \mathrm{~cm}^{-1}$ (bottom figure). The spectra presented here were recorded for a pump energy of 0.83 and $3.36 \mu \mathrm{J}$, respectively. With the chosen beam size, diffraction effects in this regime can be assumed to be negligible and the dynamics resemble a one-dimensional dynamics. Because of the simultaneous presence of phase mismatch and GVM, we simply observe in the spectra the presence of on-axis phase matching occurring at a tunable shifted frequency with respect to the exact phase matching value of $531 \mathrm{~nm}$. Note the presence of interference fringes as discussed in Secs. II A and III. The broad temporal cross-correlation pulse profile associated with

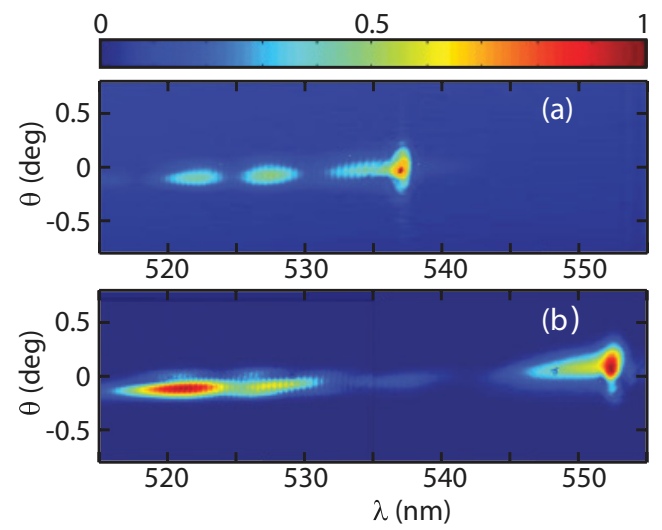

FIG. 9. (Color online) Experimental spatiotemporal spectra recorded for a fundamental pump beam of $205 \mu \mathrm{m}$ FWHM in the conditions (a) $\Delta k=23.1 \mathrm{~cm}^{-1}$ and (b) $\Delta k=55.2 \mathrm{~cm}^{-1}$.

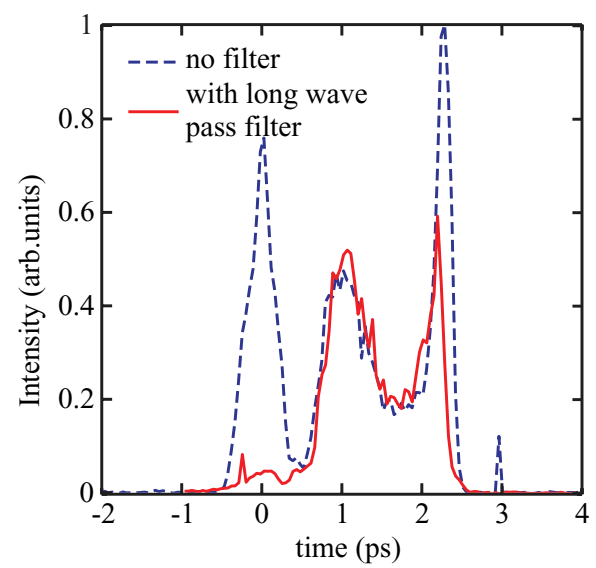

FIG. 10. (Color online) Cross-correlation profile of the shifted frequency second-harmonic radiation recorded for $\Delta k=23.1 \mathrm{~cm}^{-1}$ and a fundamental pump beam of $205 \mu \mathrm{m}$ FWHM.

the shifted second-harmonic component in the case of $\Delta k=$ $23.1 \mathrm{~cm}^{-1}$ is shown in Fig. 10. The measurement was performed by using a long-wave-pass RazorEdge filter cutting the exact $\mathrm{SH}$ component $(531 \mathrm{~nm})$.

Spatiotemporal spectral measurements in the defocusing regime have also been performed for a smaller size of the fundamental beam. Figure 11 shows three spectra recorded in three different phase-mismatch conditions in the case of a 140- $\mu \mathrm{m}$ FWHM pump; namely, $\Delta k=23.1 \mathrm{~cm}^{-1}, \Delta k=$ $33.8 \mathrm{~cm}^{-1}$, and $\Delta k=55.2 \mathrm{~cm}^{-1}$, respectively. The on-axis contributions of the second-harmonic field lie, respectively, around 539, 543, and $552 \mathrm{~nm}$, as expected. On the other hand, thanks to the angular dispersion, other frequencies are clearly matched off axis, as predicted by the theory. The bending of the tails, which can be observed in the measured spectra, depends on the sign of the GVM between the interacting

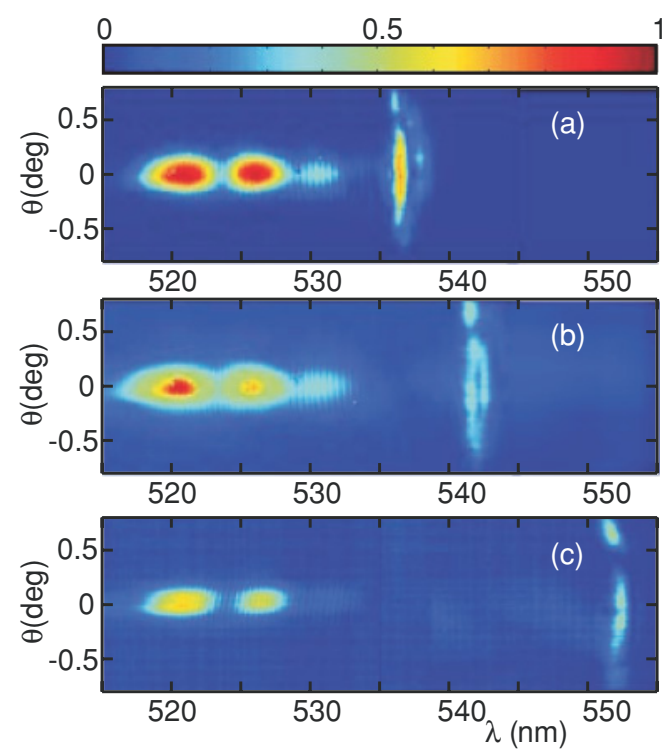

FIG. 11. (Color online) Experimental spatiotemporal spectra recorded for a fundamental pump beam of $140 \mu \mathrm{m}$ FWHM in the conditions (a) $\Delta k=23.1 \mathrm{~cm}^{-1}$, (b) $\Delta k=33.8 \mathrm{~cm}^{-1}$, and (c) $\Delta k=$ $55.2 \mathrm{~cm}^{-1}$. 


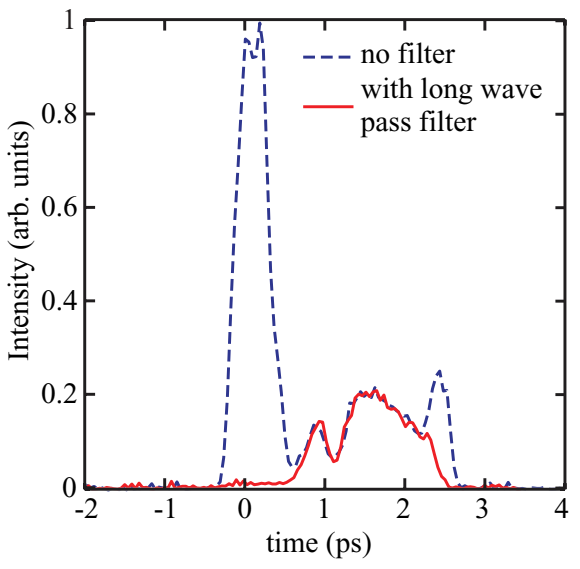

FIG. 12. (Color online) Cross-correlation profiles of the secondharmonic radiation recorded for $\Delta k=23.1 \mathrm{~cm}^{-1}$ and a fundamental pump beam of $140 \mu \mathrm{m}$ FWHM. These have been recorded with (red solid line) and without (blue dashed line) the long-wave-pass RazorEdge filter and they are, respectively, associated with the complete SH and the shifted frequency SH contribution only.

pulses. Note that self-defocusing leads to a fast divergence of the fundamental beam and thus to a small efficiency of the SH process, and higher energies here are needed to see the phase-mismatched second-harmonic generation, especially off axis. An illustration of the temporal profile of the generated SH radiation is given in Fig. 12 for the case of $\Delta k=23.1 \mathrm{~cm}^{-} 1$, where we show the cross-correlation traces recorded with (red solid line) and without (blue dashed line) the long-wave-pass RazorEdge filter. The width (FWHM) of the temporal component associated with the off-axis contribution turns out to be around $1.5 \mathrm{ps}$. This pulse (approaching a rectangular shape) is thus much longer than the delayed pulse corresponding to the exact phase-matched radiation in the second-harmonic process. Note that the broadening and the tunability of the generated phase-mismatched SH peak confirm previous observations [21]. Here we see experimentally that the shifted frequency is tunable by more than $30 \mathrm{~nm}$.

\section{B. SHG in self-focusing regime}

A clear three-dimensional dynamics of the secondharmonic generation process is observed when pumping the LBO crystal by means of a broad angular spectrum pump (i.e., with small beam width) and in the self-focusing regime. To work in these experimental conditions, the temperature of the crystal was selected to be greater than the phase-matching temperature, in such a way to have $\Delta k<0$. The pump beam width (FWHM) was $140 \mu \mathrm{m}$. In this case, we can obtain phase matching for a broad range of frequencies by generating, in addition to the exact phase-matched radiation, frequency shifted components together with evident angular dispersion. This can be seen in Fig. 13, where we report the experimental spectra (a) and (c) corresponding to two different phase-mismatch conditions, namely, $\Delta k=-17.6 \mathrm{~cm}^{-1}$ (with pump energy of $0.848 \mu \mathrm{J}$ ) and $\Delta k=-41.1 \mathrm{~cm}^{-1}$ (with pump energy of $1 \mu \mathrm{J}$ ). The on-axis phase matching occurs in these cases, respectively, around 525 and $518 \mathrm{~nm}$ as expected. The reshaping of the SH radiation is evident. In both cases, the spectrum clearly showing the formation of angular dispersion
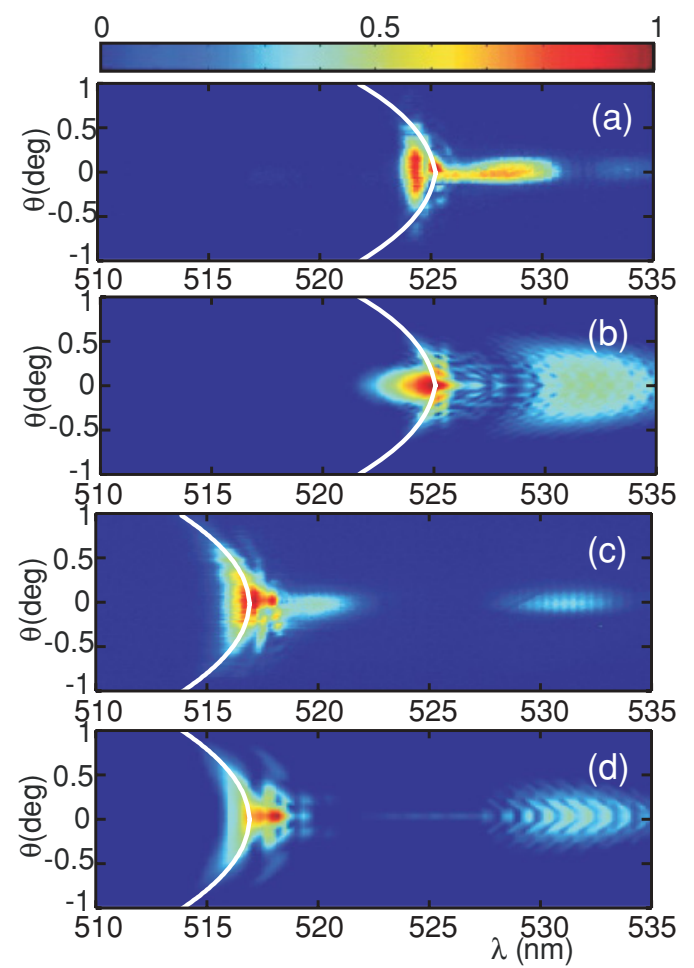

FIG. 13. (Color online) (a), (c) Experimental and (b), (d) numerically simulated spatiotemporal spectra associated with a fundamental pump beam of $140 \mu \mathrm{m}$ FWHM in the conditions of (a), (b) $\Delta k=$ $-17.6 \mathrm{~cm}^{-1}$ and (c), (d) $\Delta k=-41.1 \mathrm{~cm}^{-1}$.

[Figs. 13(a) and 13(c)] has been fitted with the X-wave spectral relation [Eq. (42)] for a wave traveling at the group velocity of the pump. A comparison with a corresponding spectrum obtained from a numerical simulation is shown in Figs. 13(b) and 13(d). Indeed, we find from the fit a group velocity of $u_{1}=1.8410^{8} \mathrm{~m} / \mathrm{s}$ in perfect accordance with the group velocity of a $1062-\mathrm{nm}$ pump pulse.

The cross-correlation profile of the SH radiation measured in the case $\Delta k=-17.6 \mathrm{~cm}^{-1}$ with a low-pass RazorEdge filter is shown in Fig. 14 (red dashed line). The corresponding

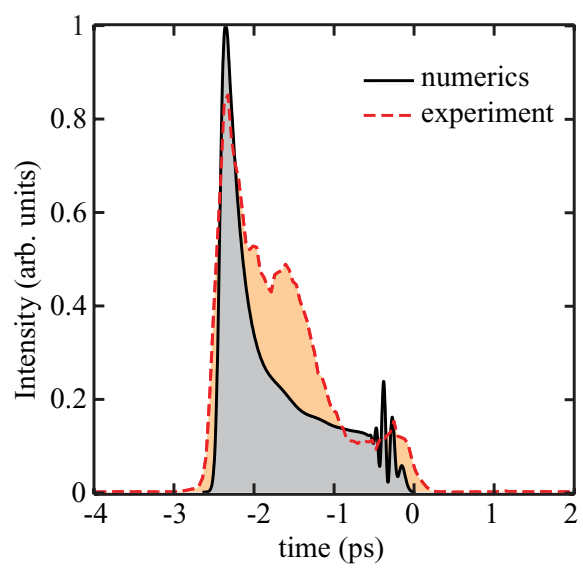

FIG. 14. (Color online) Measured second-harmonic crosscorrelation profile (red dashed line) and corresponding on-axis pulse envelope obtained by numerical simulation in the case $\Delta k=$ $-17.6 \mathrm{~cm}^{-1}$ with a fundamental pump beam of $140 \mu \mathrm{m}$ FWHM. 

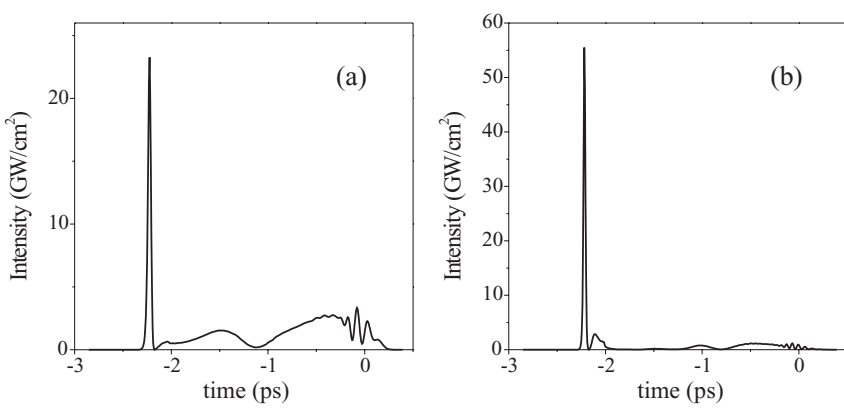

FIG. 15. The $\mathrm{SH}$ on-axis profile obtained by computer simulation with the optimized input fundamental beam wave-front curvature radius $R_{0}$. (a) $\Delta k=-17.6 \mathrm{~cm}^{-1}$ and $R_{0}=29 \mathrm{~mm}$, and (b) $\Delta k=$ $-41.1 \mathrm{~cm}^{-1}$ and $R_{0}=27 \mathrm{~mm}$.

on-axis $\mathrm{SH}$ intensity profile from numerical simulation is shown also in Fig. 14 with the black plain line. Note that, in this case, the pass-band filter we had could not cut completely the exact phase-matching contribution at $531 \mathrm{~nm}$. To obtain good agreement of the numerical data with the experimentally measured $\mathrm{SH}$ profile, the $\mathrm{FH}$ beam at the input was slightly defocused (beam wave-front radius was set at $R_{0}=23.5 \mathrm{~mm}$ ). The on-axis SH pulse FWHM duration was nearly $300 \mathrm{fs}$. Such conditions are not optimal for the shortest SH pulse formation. As it was demonstrated by numeric simulation, it is possible to generate a much shorter on-axis $\mathrm{SH}$ pulse by choosing a proper $\mathrm{FH}$ beam wave-front curvature at the input. Computer simulation results are presented in Fig. 15 in the conditions of (a) $\Delta k=-17.6 \mathrm{~cm}^{-1}$ and (b) $\Delta k=-41.1 \mathrm{~cm}^{-1}$. In the case Fig. 15(a), the on-axis SH pulse with 35 fs FWHM duration could be obtained by setting the FH beam wave-front radius to $R_{0}=29 \mathrm{~mm}$; in the case Fig. $15(\mathrm{~b})$, the SH pulse duration is $22 \mathrm{fs}$ if $R_{0}=27 \mathrm{~mm}$.

\section{v. CONCLUSIONS}

In this paper, we have presented a detailed theoretical and numerical description of the second-harmonic-generation process under phase-mismatched conditions, carefully analyzing different regimes of focusing, beam dimensions, and positive or negative phase mismatch, with the presence or absence of group-velocity mismatch and group-velocity dispersion. It has been shown that, in a sufficiently long medium, the phase-mismatched SHG becomes self-phase matched at a shifted frequency. In the case of a broad FH beam (when the plane-wave approximation is valid in the so-called 1D case), the duration of the self-phase-matched SH pulse component increases proportionally to the propagation distance and is free from angular dispersion. In the case of a narrow FH beam (the so-called 3D interaction case), the self-phase matching occurs also for off-axis components, leading to the generation of a propagation-invariant $\mathrm{X}$ pulse (with longitudinal wave-vector linearly dependent on the frequency). This short SH pulse propagates with the group velocity equal to that of the fundamental one, while keeping its duration nearly constant. Transition between the $1 \mathrm{D}$ and the $3 \mathrm{D}$ dynamics naturally occurs by tuning the phase-mismatch parameter $\Delta k$ from the self-defocusing $(\Delta k>0)$ to the self-focusing $(\Delta k<0)$ regime.

Experimental measurements performed in the femtosecond-pulsed regime in an LBO crystal in both the focusing and self-defocusing cases have confirmed the theoretical predictions, illustrating by means of a spatiotemporal diagnostics the generation at a shifted frequency of a picosecond $\mathrm{SH}$ pulse, together with its tunability, the narrowing of its corresponding spectrum, as well as the presence of angular dispersion, which is well evident in the three-dimensional case.

It is worth noting that this study can be extended to a medium with cubic nonlinearity, as the appearance of the second-harmonic $\mathrm{X}$ pulse at shifted frequency may be considered analogous to the generation of the blue-shifted component in the filamentation regime characteristics of Kerr media [39].

\section{ACKNOWLEDGMENT}

The authors wish to acknowledge the Seventh Framework Programme (LASERLAB-EUROPE CONT, Grant Agreement No. 212025).
[1] P. A. Franken, A. E. Hill, C. W. Peters, and G. Weinreich, Phys. Rev. Lett. 7, 118 (1961).

[2] J. A. Armstrong, N. Bloembergen, J. Ducuing, and P. S. Pershan, Phys. Rev. 127, 1918 (1962).

[3] N. Bloembergen and P. S. Pershan, Phys. Rev. 128, 606 (1962).

[4] P. D. Maker, R. W. Terhune, M. Nisenoff, and C. M. Savage, Phys. Rev. Lett. 8, 21 (1962).

[5] G. D. Boyd and D. A. Kleinman, J. Appl. Phys. 39, 3597 (1968).

[6] N. Bloembergen, H. J. Simon, and C. H. Lee, Phys. Rev. 181, 1261 (1969).

[7] J. Comly and E. Garmire, Appl. Phys. Lett. 12, 7 (1968).

[8] S. L. Shapiro, Appl. Phys. Lett. 13, 19 (1968).

[9] W. H. Glenn, IEEE J. Quantum Electron. 5, 6 (1969).

[10] J. T. Manassah and O. R. Cockings, Opt. Lett. 12, 1005 (1987).
[11] J. T. Manassah, Appl. Opt. 27, 4365 (1988).

[12] H. J. Bakker, P. C. M. Planken, and H. G. Muller, J. Opt. Soc. Am. B 6, 1665 (1989).

[13] N. C. Khotari and X. Charlotti, J. Opt. Soc. Am. B 5, 756 (1988).

[14] L. D. Noordam, H. J. Bakker, M. P. de Boer, and H. B. van Linden van den Heuvell, Opt. Lett. 15, 1464 (1990).

[15] R. M. Rassoul, A. Ivanov, E. Freysz, A. Ducasse, and F. Hache, Opt. Lett. 22, 268 (1997).

[16] M. Mlejnek, E. M. Wright, J. V. Moloney, and N. Bloembergen, Phys. Rev. Lett. 83, 2934 (1999).

[17] W. Su, L. Qian, H. Luo, X. Fu, H. Zhu, T. Wang, K. Beckwitt, Y. Chen, and F. Wise, J. Opt. Soc. Am. B 23, 51 (2006).

[18] V. Roppo et al., Phys. Rev. A 76, 033829 (2007). 
[19] M. Centini, V. Roppo, E. Fazio, F. Pettazzi, C. Sibilia, J. W. Haus, J. V. Foreman, N. Akozbek, M. J. Bloemer, and M. Scalora, Phys. Rev. Lett. 101, 113905 (2008).

[20] E. Fazio, F. Pettazzi, M. Centini, M. Chauvet, A. Belardini, M. Alonzo, C. Sibilia, M. Bertolotti, and M. Scalora, Opt. Express 17, 3141 (2009).

[21] M. Marangoni, D. Brida, M. Quintavalle, G. Cirmi, F. M. Pigozzo, C. Manzoni, F. Baronio, A. D. Capobianco, and G. Cerullo, Opt. Express 15, 8884 (2007).

[22] K. Moutzouris, F. Adler, F. Sotier, D. Trutlein, and A. Leitenstorfer, Opt. Lett. 31, 1148 (2006).

[23] M. Marangoni, D. Brida, M. Conforti, A. D. Capobianco, C. Manzoni, F. Baronio, G. F. Nalesso, C. De Angelis, R. Ramponi, and G. Cerullo, Opt. Lett. 34, 241 (2009).

[24] P. Di Trapani, G. Valiulis, A. Piskarskas, O. Jedrkiewicz, J. Trull, C. Conti, and S. Trillo, Phys. Rev. Lett. 91, 093904 (2003).

[25] O. Jedrkiewicz, J. Trull, G. Valiulis, A. Piskarskas, C. Conti, S. Trillo, and P. Di Trapani, Phys. Rev. E 68, 026610 (2003).

[26] W. Lei, J. Wu, H. Cai, and H. Zeng, Opt. Lett. 34, 166 (2009).

[27] M. Kolesik, E. M. Wright, and J. V. Moloney, Phys. Rev. Lett. 92, 253901 (2004).

[28] D. Faccio, P. Di Trapani, S. Minardi, A. Bramati, F. Bragheri, C. Liberale, V. Degiorgio, A. Dubietis, and A. Matijosius, J. Opt. Soc. Am. B 22, 862 (2005).

[29] D. Faccio, A. Averchi, A. Dubietis, P. Polesana, A. Piskarskas, P. Di Trapani, and A. Couairon, Opt. Lett. 32, 184 (2007).
[30] D. Faccio, A. Averchi, A. Couairon, M. Kolesik, J. V. Moloney, A. Dubietis, G. Tamosauskas, P. Polesana, A. Piskarskas, and P. Di Trapani, Opt. Express 15, 13077 (2007).

[31] C. Conti, S. Trillo, P. Di Trapani, G. Valiulis, A. Piskarskas, O. Jedrkiewicz, and J. Trull, Phys. Rev. Lett. 90, 170406 (2003).

[32] For a review, see C. Conti and S. Trillo, in Localized Waves, edited by H. E. Hernandez-Figuroa, M. Zamboni-Rached, and E. Recami (Wiley Interscience, New York, 2007), Chap. 9, p. 243.

[33] S. Trillo, C. Conti, P. Di Trapani, O. Jedrkiewicz, J. Trull, G. Valiulis, and G. Bellanca, Opt. Lett. 27, 1451 (2002).

[34] C. Conti and S. Trillo, Opt. Lett. 28, 1251 (2003).

[35] A. Yariv, Quantum Electronics (Wiley, New York, 1989).

[36] For a review, see M. Porras, P. Di Trapani, and W. Hu, in Localized Waves, edited by H. E. Hernandez-Figuroa, M. Zamboni-Rached, and E. Recami (Wiley Interscience, New York, 2007), Chap. 8, p. 217.

[37] P. Di Trapani, A. Bramati, S. Minardi, W. Chinaglia, C. Conti, S. Trillo, J. Kilius, and G. Valiulis, Phys. Rev. Lett. 87, 183902 (2001).

[38] C. Conti, S. Trillo, P. Di Trapani, J. Kilius, A. Bramati, S. Minardi, W. Chinaglia, and G. Valiulis, J. Opt. Soc. Am. B 19, 852 (2002).

[39] D. Faccio, A. Averchi, A. Lotti, M. Kolesik, J. V. Moloney, A. Couairon, and P. Di Trapani, Phys. Rev. A 78, 33825 (2008). 